\title{
THE SUFFICIENCY OF THE MATKOWSKY CONDITION IN THE PROBLEM OF RESONANCE ${ }^{1}$
}

BY

\author{
CHING-HER LIN ${ }^{2}$
}

\begin{abstract}
We consider the sufficiency of the Matkowsky condition concerning the differential equation $\varepsilon y^{\prime \prime}+f(x, \varepsilon) y^{\prime}+g(x, \varepsilon) y=0(-a \leqslant x \leqslant b)$ under the assumption that $f(0, \varepsilon)=0$ identically in $\varepsilon, f_{x}(0, \varepsilon) \neq 0$ with $f>0$ for $x<0$ and $f<0$ for $x>0$. Y. Sibuya proved that the Matkowsky condition implies resonance in the sense of $\mathrm{N}$. Kopell if $f$ and $g$ are convergent power series for $|\varepsilon|<\rho(\rho>0)$, $f(x, 0)=-2 x$ and the interval $[-a, b]$ is contained in a disc $D$ with center at 0 . The main problem in this work is to remove from Sibuya's result the assumption that $D$ is a disc.
\end{abstract}

1. Introduction. Let us consider the differential equation

$$
\varepsilon y^{\prime \prime}+f(x, \varepsilon) y^{\prime}+g(x, \varepsilon) y=0
$$

in which the independent variable $x$ ranges over a real interval $[-a, b]$ with $a, b>0$, and $\varepsilon$ is a small positive parameter. The coefficients $f(x, \varepsilon)$ and $g(x, \varepsilon)$ are continuously differentiable real-valued functions of $x$ and $\varepsilon, f(0, \varepsilon)=0$ identically in $\varepsilon$, $f_{x}(0, \varepsilon) \neq 0$ with $f>0$ for $x<0, f<0$ for $x>0$. We consider (1.1) together with boundary conditions

$$
y(-a, \varepsilon)=1, \quad y(b, \varepsilon)=B,
$$

where $B$ is a real constant. It is known that, in general, the solution $y(x, \varepsilon)$ to $(1.1)$ and (1.2) converges to zero as $\varepsilon$ tends to zero in the real interval $(-a, b)$. However, some exceptional cases may arise in which the solution $y(x, \varepsilon)$ of $(1.1)$ and (1.2) converges on $(-a, b)$ to a nontrivial solution of

$$
f(x, 0) y^{\prime}+g(x, 0) y=0 .
$$

Indeed, Ackerberg and O'Mally [2] found that unless $l=-g(0,0) / f_{x}(0,0)$ is a nonnegative integer, the solution $y(x, \varepsilon)$ of (1.1) and (1.2) converges to zero as $\varepsilon$ tends to zero, and the term "resonance" is applied to those cases when the limit of $y(x, \varepsilon)$ as $\varepsilon$ tends to zero is a nontrivial solution of (1.3).

Watts [16] considered the problem with $f(x, \varepsilon)=-x$ and $g(x, \varepsilon)=l+x$, where $l$ is a nonnegative integer, and showed that the above condition is not sufficient for resonance. Cook and Eckhaus [3] found that if $g(x, \varepsilon)=l+x+\alpha_{1} \varepsilon+\alpha_{2} \varepsilon^{2}+\cdots$,

\footnotetext{
Received by the editors June 30, 1982.

1980 Mathematics Subject Classification. Primary 34E20.

${ }^{1}$ This paper was presented in a special session on asymptotic solutions of ordinary differential equations at the April 17, 1982 meeting of the AMS at the University of Wisconsin, Madison.

${ }^{2}$ The research of the author was partially sponsored by the National Science Foundation under Grant MCS 79-01998 and Control Science Department, University of Minnesota.
} 
then $\alpha_{1}=-1$ is also a necessary condition for resonance. They predicted that for resonance we need infinitely many conditions. Therefore one of the major problems of (1.1) and (1.2) is to determine necessary and sufficient conditions for resonance to take place. B. J. Matkowsky [9] proposed the following condition:

"there exists a nontrivial formal power series in $\varepsilon$,

$$
y=\sum_{m=0}^{\infty} a_{m}(x) \varepsilon^{m},
$$

which formally satisfies (1.1) and such that all the $a_{m}(x)$ are bounded on the real interval $[-a, b]$.

N. Kopell [5] and F. Olver [10] have shown that Matkowsky's condition is necessary for resonance. A formal solution $y=\sum_{m=0}^{\infty} a_{m}(x) \varepsilon^{m}$ of (1.1) is called an outer expansion. Note that boundary conditions are not involved in the Matkowsky condition.

We say that (1.1) exhibits resonance in the sense of Kopell on $[-a, b]$ if there exists a solution $y(x, \varepsilon)$ satisfying $y(-a, \varepsilon)=1$, (disregarding $y(b, \varepsilon)=B$ ) such that $y(x, \varepsilon)$ converges uniformly on $[-a, b]$ to a nontrivial solution of (1.3) as $\varepsilon$ tends to zero.

Note. "Resonance" in this paper means "resonance in the sense of N. Kopell".

MAIN THEOREM. The Matkowsky condition implies resonance in the sense of Kopell.

Let

$$
L=\varepsilon D^{2}+f D+g \quad(D=d / d x) .
$$

Suppose there exists a nontrivial outer expansion $y=\sum_{m=0}^{\infty} a_{m}(x) \varepsilon^{m}$ whose coefficients $a_{m}(x)$ are bounded on $[-a, b]$. Set $y=\phi_{N}+u$ in (1.1), where

$$
\phi_{N}(x, \varepsilon)=\sum_{m=0}^{N} a_{m}(x) \varepsilon^{m}
$$

is a finite sum of the outer expansion. Then

$$
\varepsilon u^{\prime \prime}+f(x, \varepsilon) u^{\prime}+g(x, \varepsilon) u=-L \phi_{N}=O\left(\varepsilon^{N+1}\right) .
$$

If $u=O\left(\varepsilon^{N+1}\right)$ as $\varepsilon$ tends to zero, then (1.1) exhibits resonance on [-a,b]. However, it is very difficult to show that $u=O\left(\varepsilon^{N+1}\right)$ from (1.4). For example, let us consider

$$
\varepsilon y^{\prime \prime}-2 x y^{\prime}+(p-1) y=\psi(\varepsilon),
$$

where $p$ is a positive odd integer. The transformations

$$
y=w \exp \left(x^{2} / 2 \varepsilon\right) \text { and } x=t \varepsilon^{1 / 2}
$$

take

$$
\varepsilon y^{\prime \prime}-2 x y^{\prime}+(p-1) y=0
$$

to

$$
d^{2} w / d t^{2}-\left(t^{2}-p\right) w=0
$$


(Weber's equation, cf. [17]). P. F. Hsieh and Y. Sibuya [4] constructed the unique solution $w_{1}(t, p)$ of (1.8) such that $w_{1}(t, p)$ and $w_{1}^{\prime}(t, p)$ admit, respectively, the asymptotic representations

$$
\begin{aligned}
& w_{1}(t, p)=t^{(p-1) / 2} e^{-t^{2} / 2}\left[1+O\left(|t|^{-2}\right)\right], \\
& w_{1}^{\prime}(t, p)=t^{(p+1) / 2} e^{-t^{2} / 2}\left[-1+O\left(|t|^{-2}\right)\right]
\end{aligned}
$$

as $t$ tends to infinity in any closed subsector of the open sector: $|\arg t|<3 \pi / 4$. Let $\omega=\exp \left(\frac{1}{2} \pi i\right)$; if $t$ is replaced by $\omega^{-1} t$ and $p$ by $-p$, then (1.8) does not change. Thus $w_{1}\left(\omega^{-1} t,-p\right)$ is also a solution of (1.8) and

$$
\lim _{t \rightarrow \infty} \omega^{-(p+1) / 2} t^{(p+1) / 2} e^{-t^{2} / 2} w_{1}\left(\omega^{-1} t,-p\right)=1, \quad \text { for }\left|\arg t-\frac{\pi}{2}\right|<\frac{3 \pi}{4} .
$$

Similarly, $w_{1}\left(\omega^{-2} t, p\right)$ and $w_{1}(\omega t,-p)$ are also solutions of (1.8) with

$$
\begin{array}{ll}
\lim _{t \rightarrow \infty} \omega^{-(1-p)} t^{(1-p) / 2} e^{t^{2} / 2} w_{1}\left(\cdot^{-2} t, p\right)=1, & \text { for }|\arg t-\pi|<\frac{3 \pi}{4}, \\
\lim _{t \rightarrow \infty} \omega^{(1+p) / 2} t^{(1+p) / 2} e^{-t^{2} / 2} w_{1}(\omega t,-p)=1, & \text { for }\left|\arg t+\frac{\pi}{2}\right|<\frac{3 \pi}{4} .
\end{array}
$$

The general solution of (1.8) is

$$
w=C_{0} w_{1}(t, p)+C_{1} w_{1}\left(\omega^{-1} t,-p\right)
$$

where $C_{0}$ and $C_{1}$ are arbitrary constants. It follows from (1.6), (1.7), (1.8), (1.13) and variation of parameters that (1.5) admits a solution

$$
\begin{aligned}
y= & \exp \left(\frac{x^{2}}{2 \varepsilon}\right) w_{1}\left(\frac{x}{\sqrt{\varepsilon}}, p\right)\left\{C_{0}-\int_{0}^{x} \frac{w_{1}\left(\omega^{-1} \tau / \varepsilon^{1 / 2},-p\right) \psi(\varepsilon)}{2 \varepsilon^{1 / 2} \omega^{-(p-1) / 2} \exp \left(\tau^{2} / 2 \varepsilon\right)} d \tau\right\} \\
& +\exp \left(\frac{x^{2}}{2 \varepsilon}\right) w_{1}\left(\frac{\omega^{-1} x}{\sqrt{\varepsilon}},-p\right)\left\{C_{1}+\int_{0}^{x} \frac{w_{1}\left(\tau / \varepsilon^{1 / 2}, p\right) \psi(\varepsilon)}{2 \varepsilon^{1 / 2} \omega^{-(p-1) / 2} \exp \left(\tau^{2} / 2 \varepsilon\right)} d \tau\right\} .
\end{aligned}
$$

It follows from (1.9) and (1.10) that

$$
\begin{aligned}
y \sim & \left(\frac{x}{\sqrt{\varepsilon}}\right)^{(p-1) / 2}\left\{C_{0}-\int_{0}^{x} \frac{\omega^{p} \varepsilon^{(p-1) / 4} \psi(\varepsilon)}{2 \tau^{(p+1) / 2}} d \tau\right\} \\
& +\omega^{(1+p) / 2} \varepsilon^{(1+p) / 4} x^{-(1+p) / 2} \exp \left(\frac{x^{2}}{\varepsilon}\right) \\
& \times\left\{C_{1}+\int_{0}^{x} \frac{\tau^{(p-1) / 2} \omega^{(p-1) / 2} \psi(\varepsilon)}{2 \varepsilon^{(p+1) / 4} \exp \left(\tau^{2} / \varepsilon\right)} d \tau\right\} \text { for } x>0 .
\end{aligned}
$$

Since $\exp \left(x^{2} / \varepsilon\right)$ and $\exp \left\{\left(x^{2}-\tau^{2}\right) / \varepsilon\right\}$ are exponentially large as $\varepsilon$ tends to zero, $y$ is exponentially large as $\varepsilon$ tends to zero, even if $\psi(\varepsilon)$ in (1.5) is $\varepsilon^{N}$. Therefore we obtain $y \neq O\left(\varepsilon^{N}\right)$ as $\varepsilon$ tends to zero.

Let us assume that $f$ and $g$ in (1.1) are holomorphic in two complex variables $x$ and $\varepsilon$ in the domain $x \in D,|\varepsilon|<\rho$, where $D$ is a domain in the $x$-plane containing the real interval $[-a, b]$ and $\rho$ is a positive number. As we shall show later, the 
assumption that $f$ and $g$ are convergent power series in $\varepsilon$ for $|\varepsilon|<\rho$ is very important. For example, in the case when

$$
f(x, \varepsilon) \sim \sum_{m=0}^{\infty} f_{m}(x) \varepsilon^{m}, \quad g(x, \varepsilon) \sim \sum_{m=0}^{\infty} g_{m}(x) \varepsilon^{m}
$$

as $\varepsilon$ tends to zero and $g(x, \varepsilon)$ is not holomorphic in $\varepsilon$ at $\varepsilon=0$, even if the Matkowsky condition is satisfied, (1.1) may not exhibit resonance. Let us see a more concrete example; consider

$$
\varepsilon y^{\prime \prime}-2 x y^{\prime}+\left(2+2 e^{-\alpha / \varepsilon}\right) y=0 \quad(-a \leqslant x \leqslant a)
$$

where $\alpha>0, \varepsilon>0$ and $a>0$. Since $e^{-\alpha / \varepsilon}$ is asymptotically zero as $\varepsilon$ tends to zero, (1.14) does have an outer expansion $y=x$, i.e. $a_{0}(x)=x$ and $a_{m}(x)=0$ for $m \geqslant 1$. Hence, (1.14) satisfies the Matkowsky condition. The general solution of (1.14) is

$$
y=\exp \left(\frac{x^{2}}{2 \varepsilon}\right)\left[C_{0} w_{1}\left(\frac{x}{\varepsilon^{1 / 2}}, 3+2 e^{-\alpha / \varepsilon}\right)+C_{1} w_{1}\left(\frac{\omega^{-1} x}{\varepsilon^{1 / 2}},-3-2 e^{-\alpha / \varepsilon}\right)\right],
$$

where $C_{0}$ and $C_{1}$ are arbitrary constants. It follows from (1.9) and (1.10) that, for $x>0$,

$$
y(x, \varepsilon) \sim C_{0}\left(x / \varepsilon^{1 / 2}\right)^{1+e^{-\alpha / \varepsilon}}+C_{1} \exp \left(x^{2} / \varepsilon\right)\left(\omega^{-1} x / \varepsilon^{1 / 2}\right)^{-2-e^{-\alpha / \varepsilon}} .
$$

In order to get that $y(x, \varepsilon)$ converges uniformly for $x \in[0, a]$ as $\varepsilon$ tends to zero ( $y$ and $\varepsilon y^{\prime}$ being bounded on any closed subinterval of $\left.(-a, a)\right)$, we need to estimate $C_{0}$ and $C_{1}$. It follows from (1.15) that

$$
\begin{gathered}
C_{0}=\left\{y e ^ { x ^ { 2 } / 2 \varepsilon } \left[\frac{x}{\varepsilon} w_{1}\left(\omega^{-1} \frac{x}{\varepsilon^{1 / 2}},-3-2 e^{-\alpha / \varepsilon}\right)\right.\right. \\
\left.+\omega^{-1} \varepsilon^{-1 / 2} w_{1}^{\prime}\left(\omega^{-1} \frac{x}{\varepsilon^{1 / 2}},-3-2 e^{-\alpha / \varepsilon}\right)\right] \\
\left.-y^{\prime} e^{x^{2} / 2 \varepsilon} w_{1}\left(\omega^{-1} \frac{x}{\varepsilon^{1 / 2}},-3-2 e^{-\alpha / \varepsilon}\right)\right\} / 2 \varepsilon^{-1 / 2} \omega^{-1-e^{-\alpha / \varepsilon}} e^{x^{2} / \varepsilon}, \\
C_{1}=\left\{-y e^{x^{2} / 2 \varepsilon}\left[\frac{x}{\varepsilon} w_{1}\left(\frac{x}{\varepsilon^{1 / 2}}, 3+2 e^{-\alpha / \varepsilon}\right)\right.\right. \\
\left.+\varepsilon^{-1 / 2} w_{1}^{\prime}\left(\frac{x}{\varepsilon^{1 / 2}}, 3+2 e^{-\alpha / \varepsilon}\right)\right] \\
\left.+y^{\prime} e^{x^{2} / 2 \varepsilon} w_{1}\left(\frac{x}{\varepsilon^{1 / 2}}, 3+2 e^{-\alpha / \varepsilon}\right)\right\} / 2 \varepsilon^{-1 / 2} \omega^{-1-e^{-\alpha / \varepsilon}} e^{x^{2} / \varepsilon} ;
\end{gathered}
$$

hence $C_{0}$ and $C_{1}$ admit, respectively, the asymptotic representation

$$
\begin{gathered}
C_{0} \sim \varepsilon^{1 / 2+1 / 2 e^{-\alpha / \varepsilon}} \omega^{1+2 e^{-\alpha / \varepsilon}}\left\{y x^{-1-e^{-\alpha / \varepsilon}}+\frac{1}{2} \varepsilon y^{\prime} x^{-2-e^{-\alpha / \varepsilon}}\right\}, \\
C_{1} \sim e^{-\left(x^{2} / \varepsilon\right)} \varepsilon^{-1-(1 / 2) e^{-\alpha / \varepsilon}} \omega^{-1-e^{-\alpha / \varepsilon}}\left\{-y x^{2+e^{-\alpha / \varepsilon}}+\frac{1}{2} \varepsilon y^{\prime} x^{1+e^{-\alpha / \varepsilon}}\right\}
\end{gathered}
$$

as $\varepsilon$ tends to zero and $x \in(0, a)$; therefore we obtain

$$
C_{0}=C(\varepsilon) \varepsilon^{1 / 2}, \quad C_{1}=\tilde{C}(\varepsilon) e^{-a^{2} / \varepsilon},
$$


where $C(\varepsilon)$ is bounded and does not converge to zero as $\varepsilon$ tends to zero, and $\tilde{C}(\varepsilon)=O\left(\varepsilon^{-1}\right)$ as $\varepsilon$ tends to zero. Thus,

$$
\begin{aligned}
y=\exp \left(\frac{x^{2}}{2 \varepsilon}\right)[ & C(\varepsilon) \varepsilon^{1 / 2} w_{1}\left(x / \varepsilon^{1 / 2}, 3+2 e^{-\alpha / \varepsilon}\right) \\
& \left.+\tilde{C}(\varepsilon) e^{-a^{2} / \varepsilon} w_{1}\left(\omega^{-1} x / \varepsilon^{1 / 2},-3-2 e^{-\alpha / \varepsilon}\right)\right] .
\end{aligned}
$$

We now consider $x<0$. It is known that the function $w_{1}\left(x / \varepsilon^{1 / 2}, 3+2 e^{-\alpha / \varepsilon}\right)$ admits the following connection formulas (cf. [1, p. 687]):

$$
\begin{aligned}
w_{1}\left(x / \varepsilon^{1 / 2}, 3\right. & \left.+2 e^{-\alpha / \varepsilon}\right)=\frac{\sqrt{2 \pi} \omega^{2+e^{-\alpha / \varepsilon}}}{2^{(1 / 2)\left(3+2 e^{-\alpha / \varepsilon}\right)} \Gamma\left(-1-e^{-\alpha / \varepsilon}\right)} \\
& \times w_{1}\left(\omega^{-1} x / \varepsilon^{1 / 2},-3-2 e^{-\alpha / \varepsilon}\right)+\omega^{2+2 e^{-\alpha / \varepsilon}} w_{1}\left(\omega^{-2} x / \varepsilon^{1 / 2}, 3+2 e^{-\alpha / \varepsilon}\right) .
\end{aligned}
$$

Also, the inverse of the Gamma function, $\Gamma\left(-1-e^{-\alpha / \varepsilon}\right)$, satisfies an estimate

$$
K_{1} e^{-\alpha / \varepsilon} \leqslant 1 / \Gamma\left(-1-e^{-\alpha / \varepsilon}\right) \leqslant K_{2} e^{-\alpha / \varepsilon}
$$

for some positive numbers $K_{1}$ and $K_{2}$; hence we have

$$
\begin{aligned}
& y(x, \varepsilon)=\exp \left(\frac{x^{2}}{2 \varepsilon}\right)\left\{C(\varepsilon) \varepsilon^{1 / 2} \omega^{2+2 e^{-\alpha / \varepsilon}} w_{1}\left(\omega^{-2} x / \varepsilon^{1 / 2}, 3+2 e^{-\alpha / \varepsilon}\right)\right. \\
& +\left[\frac{\sqrt{2 \pi} \omega^{2+e^{-\alpha / \varepsilon}}}{2^{(1 / 2)\left(3+2 e^{-\alpha / \varepsilon}\right)} \Gamma\left(-1-e^{-\alpha / \varepsilon}\right)} C(\varepsilon) \varepsilon^{1 / 2}+\tilde{C}(\varepsilon) e^{-a^{2} / \varepsilon}\right] \\
& \left.\times w_{1}\left(\omega^{-1} x / \varepsilon^{1 / 2},-3-2 e^{-\alpha / \varepsilon}\right)\right\} \\
& \sim C(\varepsilon) \varepsilon^{1 / 2}\left(x / \varepsilon^{1 / 2}\right)^{1+e^{-\alpha / \varepsilon}}+\left(x / \varepsilon^{1 / 2}\right)^{-2-e^{-\alpha / \varepsilon}} \\
& \times\left[\sqrt{2 \pi} 2^{-(1 / 2)\left(3+2 e^{-\alpha / \varepsilon}\right)} \omega^{2 e^{-\alpha / \varepsilon}} C(\varepsilon) \varepsilon^{1 / 2} \exp \left(\frac{x^{2}-\alpha}{\varepsilon}\right)\right. \\
& \left.+\omega^{2+e^{-\alpha / \varepsilon}} \tilde{C}(\varepsilon) \exp \left\{-\left(\frac{a^{2}-x^{2}}{\varepsilon}\right)\right\}\right] \text {. }
\end{aligned}
$$

Note that $C(\varepsilon)$ is bounded and does not converge to zero as $\varepsilon$ tends to zero, and $\tilde{C}(\varepsilon)=O\left(\varepsilon^{-1}\right)$ as $\varepsilon$ tends to zero. If $\alpha<a^{2}$ then

$$
\sqrt{2 \pi} 2^{-\left(3+2 e^{-\alpha / \varepsilon}\right) / 2} \omega^{2 e^{-\alpha / \varepsilon}} C(\varepsilon) \varepsilon^{1 / 2} e^{\left(x^{2}-\alpha\right) / \varepsilon}+\omega^{2+e^{-\alpha / \varepsilon}} \tilde{C}(\varepsilon) e^{-\left(a^{2}-x^{2}\right) / \varepsilon}
$$

is exponentially large for $x$ near $-a$. Hence (1.14) does not exhibit a resonance if $\alpha<a^{2}$. Note that if $\alpha \geqslant a^{2}$ then (1.14) exhibits resonance on $[-a, a]$. 
Y. Sibuya [14] proved that the Matkowsky condition implies resonance if $f(x, 0)$ $=-2 x$ and the real interval $[-a, b]$ is contained in a disc $D$ with center at 0 . The main ideas in his proof are:

(1) Assume (1.1) satisfies the Matkowsky condition. After making changes of the independent variable and the unknown, (1.1) then becomes

$$
\varepsilon y^{\prime \prime}-2 x y^{\prime}+\left(p-1+\delta_{j}(\varepsilon)\right) y=0,
$$

respectively, in each of the domains

$$
x \in D, \quad \varepsilon \in S_{j}=\left\{\varepsilon: a_{j}<\arg \varepsilon<b_{j}, 0<|\varepsilon|<\rho_{0}\right\} \quad(j=1,2, \ldots, \nu),
$$

where $p$ is a positive odd integer, $S_{1} \cup S_{2} \cup \cdots \cup S_{\nu}=\left\{\varepsilon: 0<|\varepsilon|<\rho_{0}\right\}$, and

(i) $\delta_{j}(\varepsilon)$ is holomorphic in $S_{j}=\left\{\varepsilon: a_{j}<\arg \varepsilon<b_{j}, 0<|\varepsilon|<\rho_{0}\right\}$;

(ii) $\delta_{j}(\varepsilon)$ is asymptotically zero as $\varepsilon$ tends to zero in $S_{j}$;

(2) it follows from the connection formulas of Weber's equation (1.8) that

$$
\left|\delta_{j}(\varepsilon)-\delta_{k}(\varepsilon)\right| \leqslant M_{j k} \exp \left(-r^{2} /|\varepsilon|\right) \text { for } \varepsilon \in S_{j} \cap S_{k},
$$

where $M_{j k}$ is a positive number and $r$ is the radius of disc $D$. Then by a fundamental lemma in his paper, it follows that $\left|\delta_{j}(\varepsilon)\right| \leqslant H_{j} \exp \left(-r^{2} /|\varepsilon|\right)$ for $\varepsilon \in S_{j}$, where $H_{j}$ is a positive number.

(3) Since disc $D$ contains the real interval $[-a, b], r^{2} \geqslant \max \left(a^{2}, b^{2}\right)$. By explanation of the previous example, the estimates of $\delta_{j}(\varepsilon)$ are good enough for (1.1) to exhibit resonance on $[-a, b]$.

In this paper we consider the general case where $D$ is a domain in the $x$-plane containing the real interval $[-a, b]$. The main problem is to remove from Sibuya's result the assumption that $D$ is a disc. We will show that there exist transformations taking (1.1) to (1.16-j), respectively, in each of the domains (1.17). In our case, since the domain $D$ contains a disc with radius $r>0$ and center at 0 , we can obtain $\left|\delta_{j}(\varepsilon)\right| \leqslant H_{j} \exp \left(-r^{2} /|\varepsilon|\right)$ for $\varepsilon \in S_{j}$ by using Sibuya's result. However, since the radius $r$ of such a disc is small in general, these estimates of $\delta_{j}(\varepsilon)$ are not enough for resonance. We shall use cohomological methods and generalizations of the Phragmén-Lindelöf theorem to improve the estimates of $\delta_{j}(\varepsilon)$. Then we shall show that (1.1) actually exhibits resonance on $[-a, b]$. Information is also taken from the paper by Kreiss and Parter [6].

2. Preliminaries. If we change $y$ by

$$
y=w \exp \left\{-\frac{\varepsilon}{2} \int_{0}^{x}\left[-2 \tau+f_{1}(\tau, \varepsilon)\right] d \tau\right\},
$$

where $f_{1}(\tau, \varepsilon)=\sum_{m=1}^{\infty} f_{m}(\tau) \varepsilon^{m},(1.1)$ is reduced to

$$
\varepsilon^{2} w^{\prime \prime}-\left[x^{2}+\varepsilon R(x, \varepsilon)\right] w=0,
$$

where

$$
x^{2}+\varepsilon R(x, \varepsilon)=\frac{f(x, \varepsilon)^{2}}{4}+\varepsilon\left[\frac{1}{2} \frac{\partial f}{\partial x}(x, \varepsilon)-g(x, \varepsilon)\right], \quad R(x, \varepsilon)=\sum_{m=0}^{\infty} R_{m}(x) \varepsilon^{m}
$$

and each $R_{m}(x)$ is holomorphic in the domain $D$. 
(2.1) is equivalent to the system

$$
\varepsilon d W / d x=M(x, \varepsilon) W,
$$

where

$$
W=\left[\begin{array}{c}
w \\
\varepsilon d w / d x
\end{array}\right], \quad M(x, \varepsilon)=\left[\begin{array}{cc}
0 & 1 \\
x^{2}+\varepsilon R(x, \varepsilon) & 0
\end{array}\right] .
$$

To prove the main theorem, the following two lemmas are needed, and for their proofs we refer to [14].

Lemma 2.1. Let $0 \in \Omega$ be a simply connected domain in $D$. Then there exists a two-by-two matrix

$$
\begin{aligned}
T(x, \varepsilon) & =\sum_{h=0}^{\infty} T_{h}(x) \varepsilon^{h} \\
& =\left[\begin{array}{cc}
A(x, \varepsilon) & B(x, \varepsilon) \\
\varepsilon A^{\prime}(x, \varepsilon)+\left(x^{2}+\varepsilon C(\varepsilon)\right) B(x, \varepsilon) & A(x, \varepsilon)+\varepsilon B^{\prime}(x, \varepsilon)
\end{array}\right],
\end{aligned}
$$

where $A(x, \varepsilon)=\sum_{h=0}^{\infty} A_{h}(x) \varepsilon^{h}, B(x, \varepsilon)=\sum_{h=0}^{\infty} B_{h}(x) \varepsilon^{h}$ and $C(\varepsilon)=\sum_{h=0}^{\infty} a_{h} \varepsilon^{h}$. The components of $T(x, \varepsilon)$ are formal power series in $\varepsilon$ such that:

(i) the components of the two-by-two matrices $T_{h}(x)$ are holomorphic in $\Omega$;

(ii) det $T_{0}(x)=A_{0}(x)^{2}-\left(x B_{0}(x)\right)^{2}=1$ in domain $\Omega$;

(iii) the formal transformation $W=T(x, \varepsilon) V$ reduces $(2.2)$ to

$$
\varepsilon V^{\prime}=N(x, \varepsilon) V
$$

where

$$
N(x, \varepsilon)=\left[\begin{array}{cc}
0 & 1 \\
x^{2}+\varepsilon C(\varepsilon) & 0
\end{array}\right], \quad V=\left[\begin{array}{c}
v \\
\varepsilon v^{\prime}
\end{array}\right]
$$

in the domain $\Omega$, and

$$
R_{0}(0)=a_{0}
$$

LEMMA 2.2. (1.1) satisfies the Matkowsky condition iff $a_{0}(c f .(2.5))$ is a negative odd integer and $a_{m}=0(m \geqslant 1)$.

In order to obtain our main theorem, we will introduce the following notation for a domain in the $x$-plane.

Let $r_{3}$ be a sufficiently small positive number and $r_{1}, r_{2}$ positive numbers with $r_{1}>b, r_{2}>a$, respectively. Set

$$
D_{1}=\left\{x=x_{1}+i x_{2}:-r_{3}<x_{2}<r_{3},-\sqrt{r_{2}^{2}-x_{2}^{2}}<x_{1}<\sqrt{r_{1}^{2}-x_{2}^{2}}\right\}
$$

(cf. Figure 2.1). Then $D_{1}$ is a simply connected domain in the complex $x$-plane which contains the real interval $I$. We can choose $r_{1}, r_{2}$ and $r_{3}$ so that $D_{1} \subset D$.

The main theorem will follow immediately, once the following uniform simplification is proved. 


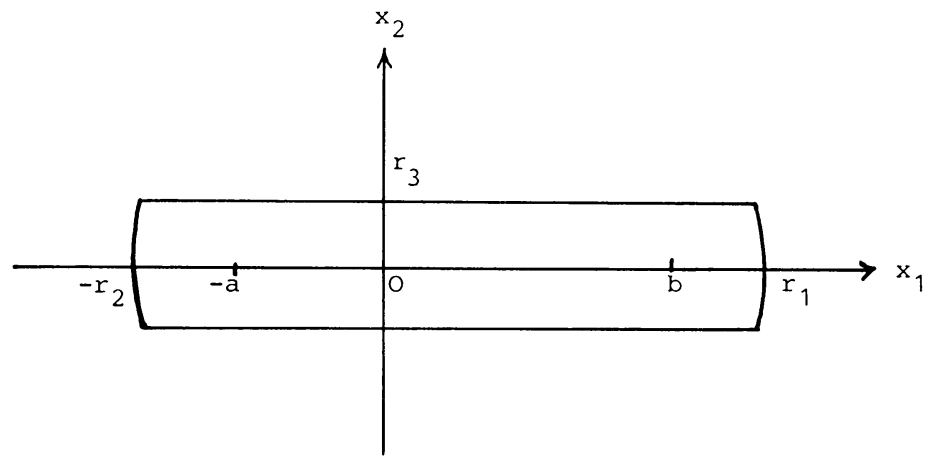

FIGURE 2.1

THEOREM 2.3. Let $\lambda$ and $M$ be sufficiently small positive numbers and set

$$
D_{2}=D_{1} \backslash\left\{x=r e^{i \theta} \in D_{1}:-3 \pi / 4-\lambda \leqslant \theta \leqslant-\pi / 4+\lambda\right\}
$$

(cf. Figure 2.2). Then there exists a two-by-two matrix $S(x, \varepsilon)$ whose components are holomorphic in two variables, $x$ and $\varepsilon$, in the domain

$$
x \in D_{1} \text {, }
$$

$$
\{\varepsilon:|\arg \varepsilon|<\lambda, 0<|\varepsilon|<M\}
$$

such that:

(i) the matrix $S(x, \varepsilon) \sim T(x, \varepsilon)(c f .(2.3))$ as $\varepsilon$ tends to zero in (2.9) uniformly in the domain (2.7);

(ii) the transformation $W=S(x, \varepsilon) U$ reduces $(2.2)$ to

$$
\varepsilon U^{\prime}=\left[\begin{array}{cc}
0 & 1 \\
x^{2}-\varepsilon p & 0
\end{array}\right] U
$$

in the domain (2.8), (2.9).

We shall prove this theorem in $§ 4$.C.

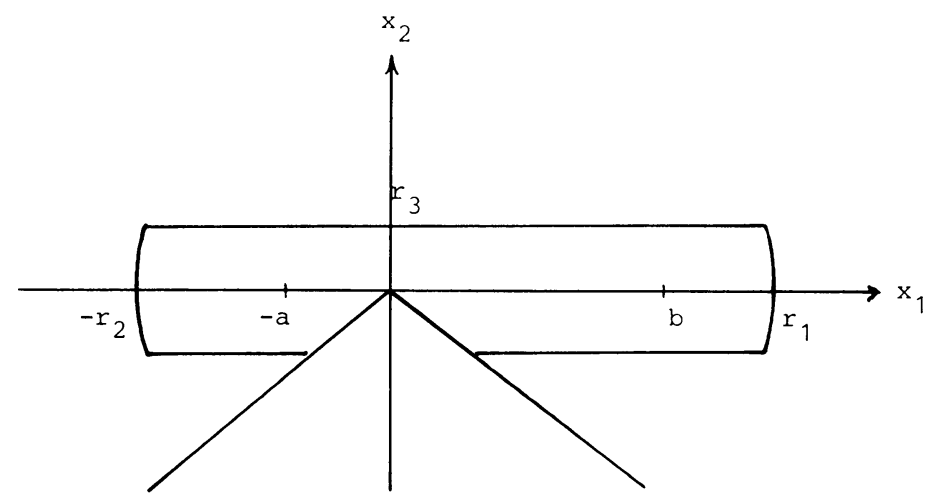

Figure 2.2

3. Basic lemmas. Assume that (1.1) satisfies the Matkowsky condition on $I$. By virtue of Lemma 2.2, this is equivalent to assuming that $a_{0}=-p$, where $p$ is a positive odd integer and $a_{m}=0$ for $m \geqslant 1$. Hereafter, we shall assume that $a \leqslant b$ 
(otherwise we replace $x$ by $-x$ ). Manipulating with rotations of domain $D_{1}$ and sectors in the $\varepsilon$-plane, we can obtain the following lemma (cf. $[8,14])$.

LEMma 3.1. There exist sectors $S_{j}=\left\{\varepsilon: a_{j}^{*}<\arg \varepsilon<b_{j}^{*}, 0<|\varepsilon|<\rho\right\} \quad(j=$ $1,2, \ldots, n)$, where $\rho$ is a positive number, $a^{*}$ 's and $b^{*}$ 's are real numbers, and $S_{1} \cup S_{2} \cup \cdots \cup S_{n}=\{\varepsilon: 0<|\varepsilon|<\rho\}$, functions $\delta_{1}(\varepsilon), \ldots, \delta_{n}(\varepsilon)$ and two-by-two matrices $P_{1}(x, \varepsilon), \ldots, P_{n}(x, \varepsilon)$ such that:

(i) $\delta_{j}(\varepsilon)$ is holomorphic in $S_{j}$;

(ii) $\delta_{j}(\varepsilon)$ is asymptotically zero as $\varepsilon$ tends to zero in $S_{j}$;

(iii) entries of $P_{j}$ and $P_{j}^{-1}$ are holomorphic in the domain

$x \in D_{1}=\left\{x=x_{1}+i x_{2}:-r_{3}<x_{2}<r_{3},-\sqrt{r_{2}^{2}-x_{2}^{2}}<x_{1}<\sqrt{r_{1}^{2}-x_{2}^{2}}\right\}, \quad \varepsilon \in S_{j}$, for some domain $D_{1}$ in $D$ which contains the real interval $I$;

(iv) $P_{j}\left(\right.$ resp. $\left.P_{j}^{-1}\right)$ admits the matrix $T$ (resp. $T^{-1}$ ) as an asymptotic expansion as $\varepsilon$ tends to zero in $S_{j}$, which is valid uniformly in $D_{1}$;

(v) the transformation $W=P_{j}(x, \varepsilon) V_{j}$ reduces $(2.2)$ to

$$
\varepsilon V_{j}^{\prime}=\left[\begin{array}{cc}
0 & 1 \\
x^{2}-\varepsilon\left(p+\delta_{j}(\varepsilon)\right) & 0
\end{array}\right] V_{j}
$$

in the domain (3.1-j).

Sibuya [14] proved that the property "asymptotically zero" of $\delta_{j}(\varepsilon)$ can be replaced by "exponentially small". In his case the domain of $x$ is a disc with center at 0 and the exponent of $e$ depends on the radius of the disc. In our case, although the domain of $x$ contains a disc with center at 0 , the radius of the disc is small. If we apply his theorem directly, we obtain

$$
\left|\delta_{j}(\varepsilon)\right| \leqslant H_{1} \exp \left(-r_{3}^{2} /|\varepsilon|\right) \quad \text { in } S_{j}, \quad j=1,2, \ldots, n,
$$

for some positive number $H_{1}$.

Let $r_{0}$ be a positive number with $a<r_{0}<r_{2}$ and choose $\omega_{0}$ with $0<\omega_{0}<\frac{1}{2} \pi$ such that $r_{3}^{2} \geqslant r_{0}^{2} \cos \omega_{0}>0$. Then if $\arg \varepsilon=\omega_{0}$ (or $-\omega_{0}$ ), we get $r_{3}^{2} /|\varepsilon| \geqslant r_{0}^{2} \operatorname{Re}(1 / \varepsilon)$. Therefore, for some sector which contains the line segment $\arg \varepsilon=\omega_{0}$ (or $-\omega_{0}$ ), $0<|\varepsilon|<\beta$, say $S_{j}$, we have

$$
\left|\delta_{j}(\varepsilon)\right| \leqslant H_{1} \exp \left\{-r_{0}^{2} \operatorname{Re}(1 / \varepsilon)\right\}
$$

for $\varepsilon$ on the line segment $\arg \varepsilon=\omega_{0}$ (or $-\omega_{0}$ ), $0<|\varepsilon|<\rho$. Then we obtain

LEMMA 3.2. There exist sectors

$$
S_{j}=\left\{\varepsilon: a_{j}<\arg \varepsilon<b_{j}, 0<|\varepsilon|<\rho\right\} \quad(j=1,2, \ldots, \nu)
$$

where

$$
-\frac{1}{2} \pi<-\omega_{0}=a_{1}<a_{2}<b_{1}<a_{3}<b_{2}<a_{4}<b_{3}<\cdots<a_{\nu}<b_{\nu-1}<b_{\nu}=\omega_{0}<\frac{1}{2} \pi,
$$


functions $\delta_{1}(\varepsilon), \ldots, \delta_{\nu}(\varepsilon)$ and two-by-two matrices $P_{1}(x, \varepsilon), \ldots, P_{\nu}(x, \varepsilon)$ such that:

(i) $\delta_{j}(\varepsilon)$ is holomorphic in $S_{j}$ and continuous on

$$
S_{j}^{*}=\left\{\varepsilon: a_{j} \leqslant \arg \varepsilon \leqslant b_{j}, 0<|\varepsilon| \leqslant \rho\right\} ;
$$

(ii) $\delta_{j}(\varepsilon)$ is asymptotically zero as $\varepsilon$ tends to zero in $S_{j}$;

(iii) $\left|\delta_{1}(\varepsilon)\right| \leqslant H_{1} \exp \left\{-r_{0}^{2} \operatorname{Re}(1 / \varepsilon)\right\}$ on the line segment $\arg \varepsilon=-\omega_{0}, 0<|\varepsilon|<\rho$; $\left|\delta_{\nu}(\varepsilon)\right| \leqslant H_{1} \exp \left\{-r_{0}^{2} \operatorname{Re}(1 / \varepsilon)\right\}$ on the line segment $\arg \varepsilon=\omega_{0}, 0<|\varepsilon|<\rho$;

(iv) entries of $P_{j}$ and $P_{j}^{-1}$ are holomorphic in the domain (3.1-j);

(v) $P_{j}\left(\right.$ resp. $\left.P_{j}^{-1}\right)$ admits the matrix $T$ (resp. $T^{-1}$ ) as an asymptotic expansion as $\varepsilon$ tends to zero in $S_{j}$, which is valid uniformly in $D_{1}$;

(vi) the transformation $W=P_{j}(x, \varepsilon) V_{j}$ reduces $(2.2)$ to $(3.1)^{\prime}$ in the domain $(3.1-j)$.

Note that these functions $\delta_{j}(\varepsilon)$ and sectors $S_{j}$ are similar to, but slightly different from, those in Lemma 3. Assumption (3.3) means that $\nu$ sectors, $S_{1}, S_{2}, \ldots, S_{\nu}$, intersect consecutively (i.e. $S_{j} \cap S_{j+1} \neq \varnothing$ ), but no three of them intersect.

The estimate of $\delta_{1}(\varepsilon)$ (resp. $\delta_{\nu}(\varepsilon)$ ) is relatively poor on the ray $\arg \varepsilon=\omega_{0}$ (resp. $\arg \varepsilon=-\omega_{0}$ ) which is close to the imaginary axis. However, we can substantially improve such an estimate along the real axis by utilizing

TheOREM 3.3. Let $S_{j}$ and $\delta_{j}$ be as in Lemma 3.2(i), (ii). Assume (3.3) holds and

$$
\left|\delta_{j+1}(\varepsilon)-\delta_{j}(\varepsilon)\right| \leqslant H_{1} \exp \{-\mu \operatorname{Re}(1 / \varepsilon)\} \quad \text { in } S_{j} \cap S_{j+1} ;
$$

$\left|\delta_{1}(\varepsilon)\right| \leqslant H_{1} \exp \{-\mu \operatorname{Re}(1 / \varepsilon)\}$ on the line segment $\arg \varepsilon=-\omega_{0}, 0<|\varepsilon|<\rho ;\left|\delta_{\nu}(\varepsilon)\right| \leqslant$ $H_{1} \exp \{-\mu \operatorname{Re}(1 / \varepsilon)\}$ on the line segment $\arg \varepsilon=\omega_{0}, 0<|\varepsilon|<\rho$, for some positive numbers $\mu$ and $H_{1}$.

Then there exists a positive number $H$ such that

$$
\left|\delta_{j}(\varepsilon)\right| \leqslant H \exp \{-\mu \operatorname{Re}(1 / \varepsilon)\} \quad \text { in } S_{j}, \quad j=1,2, \ldots, \nu .
$$

Proof. See Lin [7].

REMARK. We shall use this theorem with $\mu=r_{0}^{2}$. Note that if $\arg \varepsilon=0$ (i.e. $\varepsilon$ is positive real) and $\varepsilon \in S_{j_{0}}$ for some $j_{0}$, then (3.4) implies $\left|\delta_{j_{0}}(\varepsilon)\right| \leqslant H \exp \{-\mu / \varepsilon\}$.

\section{Proof of main theorem.}

A. Preliminaries. In this section, as an application of Theorem 3.3, we shall derive an estimate

$$
\left|\delta_{j}(\varepsilon)\right| \leqslant H \exp \left\{-r_{0}^{2} \operatorname{Re}(1 / \varepsilon)\right\} \quad \text { for } \varepsilon \in S_{j},
$$

for $\delta_{j}$ and $r_{0}$ as in Lemma 3.2. (If necessary we shall replace $\rho$ by $\tilde{\rho}$ where $0<\tilde{\rho}<\rho$.) To do this it sufficies to prove the estimate

$$
\left|\delta_{j+1}(\varepsilon)-\delta_{j}(\varepsilon)\right| \leqslant H_{1} \exp \left\{-r_{0}^{2} \operatorname{Re}(1 / \varepsilon)\right\} \quad \text { in } S_{j} \cap S_{j+1} .
$$

Note that we already have

$$
\left|\delta_{1}(\varepsilon)\right| \leqslant H_{1} \exp \left\{-r_{0}^{2} \operatorname{Re}(1 / \varepsilon)\right\}
$$

on the line segment $\arg \varepsilon=-\omega_{0}, 0<|\varepsilon|<\rho$, and

$$
\left|\delta_{\nu}(\varepsilon)\right| \leqslant H_{1} \exp \left\{-r_{0}^{2} \operatorname{Re}(1 / \varepsilon)\right\}
$$


on the line segment arg $\varepsilon=\omega_{0}, 0<|\varepsilon|<\rho$, for some positive number $H_{1}$. To derive (4.2) we shall use

$$
d^{2} w / d t^{2}-\left(t^{2}-\alpha\right) w=0
$$

(Weber's equation, cf. [17]), where $\alpha$ is a complex parameter. Hsieh and Sibuya [4] constructed the unique solution $w_{1}=w_{1}(t, \alpha)$ of (4.5) such that:

(i) $w_{1}(t, \alpha)$ is an entire function of $(t, \alpha)$;

(ii) $w_{1}(t, \alpha)$ and $w_{1}^{\prime}(t, \alpha)$ admit, respectively, the asymptotic representations

$$
\begin{aligned}
& w_{1}(t, \alpha)=t^{(\alpha-1) / 2} e^{-t^{2} / 2}\left[1+O\left(|t|^{-2}\right)\right], \\
& w_{1}^{\prime}(t, \alpha)=t^{(\alpha+1) / 2} e^{-t^{2} / 2}\left[-1+O\left(|t|^{-2}\right)\right]
\end{aligned}
$$

uniformly on each compact set in the $\alpha$-space as $t$ tends to infinity in any closed subsector of the open sector $|\arg t|<3 \pi / 4$. (Also see Y. Sibuya [12].)

Definition 4.1. If a solution of (4.5) tends to zero as $t$ tends to infinity along any direction in the sector $S$, then this solution is said to be subdominant in the sector $S$.

Since $\operatorname{Re}\left(t^{2}\right)>0$ in the sector $|\arg t|<\pi / 4$, the solution $w_{1}(t, \alpha)$ of (4.5) is called a subdominant solution in the sector $|\arg t|<\pi / 4$.

Let

$$
\omega=\exp \left(\frac{1}{2} \pi i\right)
$$

If we replace $t$ by $\omega^{-1} t$ and $\alpha$ by $-\alpha$, then (4.5) does not change. Thus $w_{1}\left(\omega^{-1} t,-\alpha\right)$ is also a solution of (4.5), and

$$
\lim _{t \rightarrow \infty} \omega^{-(\alpha+1) / 2} t^{(\alpha+1) / 2} e^{-t^{2} / 2} w_{1}\left(\omega^{-1} t,-\alpha\right)=1
$$

uniformly in $\alpha$ if $\alpha$ is in a compact set in the $\alpha$-plane and $|\arg t-\pi / 2|<3 \pi / 4$. Similarly, $w_{1}\left(\omega^{-2} t, \alpha\right)$ and $w_{1}(\omega t,-\alpha)$ are also solutions of (4.5) with

$$
\begin{gathered}
\lim _{t \rightarrow \infty} \omega^{-(1-\alpha)} t^{(1-\alpha) / 2} e^{t^{2} / 2} w_{1}\left(\omega^{-2} t, \alpha\right)=1, \\
\lim _{t \rightarrow \infty} \omega^{(1+\alpha) / 2} t^{(1+\alpha) / 2} e^{-t^{2} / 2} w_{1}(\omega t,-\alpha)=1
\end{gathered}
$$

uniformly in $\alpha$ if $\alpha$ is in a compact set in the $\alpha$-plane and $|\arg t-\pi|<3 \pi / 4$, $|\arg t+\pi / 2|<3 \pi / 4$, respectively.

(4.5) is equivalent to the system

$$
\frac{d \Psi}{d t}=\left[\begin{array}{cc}
0 & 1 \\
t^{2}-\alpha & 0
\end{array}\right] \Psi
$$

where

$$
\Psi=\left[\begin{array}{c}
w \\
d w / d t
\end{array}\right]
$$

Set

$$
\Psi_{0}(t, \alpha)=\left[\begin{array}{cc}
w_{1}(t, \alpha) & w_{1}\left(\omega^{-1} t,-\alpha\right) \\
\frac{\partial w_{1}(t, \alpha)}{\partial t} & \omega^{-1} \frac{\partial w_{1}\left(\omega^{-1} t,-\alpha\right)}{\partial t}
\end{array}\right]
$$




$$
\begin{aligned}
& \Psi_{1}(t, \alpha)=\left[\begin{array}{cc}
w_{1}\left(\omega^{-1} t,-\alpha\right) & w_{1}\left(\omega^{-2} t, \alpha\right) \\
\omega^{-1} \frac{\partial w_{1}\left(\omega^{-1} t,-\alpha\right)}{\partial t} & \omega^{-2} \frac{\partial w_{1}\left(\omega^{-2} t, \alpha\right)}{\partial t}
\end{array}\right], \\
& \Psi_{2}(t, \alpha)=\left[\begin{array}{cc}
w_{1}\left(\omega^{-2} t, \alpha\right) & w_{1}(\omega t,-\alpha) \\
\omega^{-2} \frac{\partial w_{1}\left(\omega^{-2} t, \alpha\right)}{\partial t} & \omega \frac{\partial w_{1}(\omega t,-\alpha)}{\partial t}
\end{array}\right] \text {, }
\end{aligned}
$$

and

$$
\text { (4.8-(-1)) } \quad \Psi_{-1}(t, \alpha)=\left[\begin{array}{cc}
w_{1}(\omega t,-\alpha) & w_{1}(t, \alpha) \\
\omega \frac{\partial w_{1}(\omega t,-\alpha)}{\partial t} & \frac{\partial w_{1}(t, \alpha)}{\partial t}
\end{array}\right] .
$$

We consider these matrices of independent solutions of (4.7) in $J_{0}, J_{1}, J_{2}$ and $J_{-1}$, respectively, where

$$
\begin{array}{ll}
J_{0}=\{t:|\arg t-\pi / 4|<\pi / 2\}, & J_{1}=\{t:|\arg t-\pi / 4-\pi / 2|<\pi / 2\}, \\
J_{2}=\{t:|\arg t-\pi / 4-\pi|<\pi / 2\}, & J_{-1}=\{t:|\arg t-\pi / 4+\pi / 2|<\pi / 2\} .
\end{array}
$$

Set

$$
\begin{gathered}
C(\alpha)=2^{-\alpha / 2} \omega^{(1+\alpha) / 2} \sqrt{2 \pi} / \Gamma^{(1 / 2-\alpha / 2)}, \\
\tilde{C}(\alpha)=\omega^{\alpha+3},
\end{gathered}
$$

and

$$
M(\alpha)=\left[\begin{array}{ll}
C(\alpha) & 1 \\
\tilde{C}(\alpha) & 0
\end{array}\right]
$$

Then

$$
\begin{aligned}
& \Psi_{0}(t, \alpha)=\Psi_{1}(t, \alpha) M(\alpha), \quad \Psi_{1}(t, \alpha)=\Psi_{2}(t, \alpha) M(-\alpha), \\
& \Psi_{2}(t, \alpha)=\Psi_{-1}(t, \alpha) M(\alpha), \quad \Psi_{-1}(t, \alpha)=\Psi_{0}(t, \alpha) M(-\alpha) .
\end{aligned}
$$

(Cf. Y. Sibuya [14].)

Now we shall derive solutions of the system

$$
\varepsilon V_{j}^{\prime}=\left[\begin{array}{cc}
0 & 1 \\
x^{2}-\varepsilon\left(p+\delta_{j}(\varepsilon)\right) & 0
\end{array}\right] V_{j} .
$$

Choose a branch of $\varepsilon^{1 / 2}$ such that $\arg \left(\varepsilon^{1 / 2}\right)=\frac{1}{2} \arg \varepsilon$ for $\varepsilon$ in the sector $-\omega_{0}<\arg \varepsilon$ $<\omega_{0}$. Set

$$
\Lambda(\varepsilon)=\left[\begin{array}{cc}
1 & 0 \\
0 & \varepsilon^{1 / 2}
\end{array}\right]
$$

and

$$
\Phi_{j h}(x, \varepsilon)=\Lambda(\varepsilon) \Psi_{h}\left(x / \varepsilon^{1 / 2}, p+\delta_{j}(\varepsilon)\right) \quad(h=-1,0,1,2) .
$$


Then

$$
\begin{aligned}
\varepsilon \frac{d}{d x} \Phi_{j h}(x, \varepsilon) & =\varepsilon \Lambda(\varepsilon) \frac{d}{d t} \Psi_{h}\left(\frac{x}{\varepsilon^{1 / 2}}, p+\delta_{j}(\varepsilon)\right) \varepsilon^{-1 / 2} \\
& =\varepsilon^{1 / 2} \Lambda(\varepsilon)\left[\begin{array}{cc}
0 & 1 \\
\varepsilon^{-1} x^{2}-\left(p+\delta_{j}(\varepsilon)\right) & 0
\end{array}\right] \Lambda(\varepsilon)^{-1} \Phi_{j h}(x, \varepsilon) \\
& =\left[\begin{array}{cc}
0 & 1 \\
x^{2}-\varepsilon\left(p+\delta_{j}(\varepsilon)\right) & 0
\end{array}\right] \Phi_{j h}(x, \varepsilon) .
\end{aligned}
$$

Hence, $\Phi_{j h}$ are fundamental matrix solutions of (4.12-j) such that

$$
\begin{aligned}
\Phi_{j 0}(x, \varepsilon) & =\Phi_{j 1}(x, \varepsilon) M\left(p+\delta_{j}(\varepsilon)\right), \\
\Phi_{j 1}(x, \varepsilon) & =\Phi_{j 2}(x, \varepsilon) M\left(-p-\delta_{j}(\varepsilon)\right), \\
\Phi_{j 2}(x, \varepsilon) & =\Phi_{j(-1)}(x, \varepsilon) M\left(p+\delta_{j}(\varepsilon)\right), \\
\Phi_{j(-1)}(x, \varepsilon) & =\Phi_{j 0}(x, \varepsilon) M\left(-p-\delta_{j}(\varepsilon)\right) .
\end{aligned}
$$

Set

$$
J=\left[\begin{array}{cc}
1 & 0 \\
0 & -1
\end{array}\right]
$$

and

$$
Q_{j h}(x, \varepsilon)=\Phi_{j h}(x, \varepsilon) \exp \left\{(-1)^{h} \frac{1}{2} x^{2} \varepsilon^{-1} J\right\} \quad(h=-1,0,1,2) .
$$

Some of the properties of $Q_{j h}$ are given in the following lemmas due to Sibuya [12].

LEMMA 4.2. Let $R$ and $M_{0}$ be arbitrary but fixed positive numbers and $\lambda$ a sufficiently small positive number. Then $w=w_{1}\left(\omega^{-h} x / \varepsilon^{1 / 2}, \omega^{-2 h} \alpha\right)$ satisfies

$$
\begin{aligned}
& \left|w_{1}\left(\omega^{-h} x / \varepsilon^{1 / 2}, \omega^{-2 h} \alpha\right) \exp \left\{(-1)^{h} x^{2} / 2 \varepsilon\right\}\right| \leqslant \tilde{c}|\varepsilon|^{q}, \\
& \left|w_{1}^{\prime}\left(\omega^{-h} x / \varepsilon^{1 / 2}, \omega^{-2 h} \alpha\right) \exp \left\{(-1)^{h} x^{2} / 2 \varepsilon\right\}\right| \leqslant \tilde{c}|\varepsilon|^{q}
\end{aligned}
$$

in the domain

$$
|x|<R, \quad\left|\arg \left(x / \varepsilon^{1 / 2}\right)-\frac{1}{2} h \pi\right| \leqslant 3 \pi / 4-\lambda, \quad 0<|\varepsilon|<M_{0}, \quad|\arg \varepsilon| \leqslant \omega_{0},
$$

where $\tilde{c}$ is a positive constant depending on $\lambda$, and $q$ is a real number.

LEMMA 4.3. Let $r, R$ and $M_{0}$ be arbitrary but fixed positive numbers and $\lambda a$ sufficiently small positive number. Suppose $\psi(\varepsilon)$ is a given function of $\varepsilon$ which is holomorphic in a sector

$$
\left\{\varepsilon: \rho_{1} \leqslant \arg \varepsilon \leqslant \rho_{2}, 0<|\varepsilon|<M_{0}\right\},
$$

where $-\omega_{0} \leqslant \rho_{1}<\rho_{2} \leqslant \omega_{0}$ and

$$
\psi(\varepsilon) \sim 0
$$


as $\varepsilon$ tends to zero in (4.16). Then

$$
\begin{aligned}
& {\left[w_{1}\left(\omega^{-h} \frac{x}{\varepsilon^{1 / 2}}, \omega^{-2 h} \alpha+\psi(\varepsilon)\right)-w_{1}\left(\omega^{-h} \frac{x}{\varepsilon^{1 / 2}}, \omega^{-2 h} \alpha\right)\right] \exp \left\{(-1)^{h} \frac{x^{2}}{2 \varepsilon}\right\} \sim 0,} \\
& {\left[w_{1}^{\prime}\left(\omega^{-h} \frac{x}{\varepsilon^{1 / 2}}, \omega^{-2 h} \alpha+\psi(\varepsilon)\right)-w_{1}^{\prime}\left(\omega^{-h} \frac{x}{\varepsilon^{1 / 2}}, \omega^{-2 h} \alpha\right)\right] \exp \left\{(-1)^{h} \frac{x^{2}}{2 \varepsilon}\right\} \sim 0}
\end{aligned}
$$

uniformly for

$$
|x| \leqslant R, \quad\left|\arg \left(x / \varepsilon^{1 / 2}\right)-h \pi / 2\right| \leqslant 3 \pi / 4-\lambda, \quad|\alpha| \leqslant r,
$$

as $\varepsilon$ tends to zero in the sector (4.16).

Given any $\varepsilon$ in $S_{j}$, by Lemmas 4.2 and 4.3 we know that if $(x, \varepsilon)$ is in a domain

$$
x \in D_{1}, \quad\left|\arg \left(x / \varepsilon^{1 / 2}\right)-\pi / 4-h \pi / 2\right| \leqslant \pi / 2-\lambda,
$$

where $\lambda$ is a small positive number, then we have

$$
\left\|Q_{j h}(x, \varepsilon)\right\| \leqslant \tilde{c}|\varepsilon|^{q}, \quad\left\|Q_{j h}(x, \varepsilon)^{-1}\right\| \leqslant \tilde{c}|\varepsilon|^{q},
$$

where $\tilde{c}$ is a positive number depending on $\lambda, q$ is a real number and \|\| denotes the usual matrix norm. Furthermore, the matrix $Q_{j h}(x, \varepsilon)-Q_{j+1, h}(x, \varepsilon)$ is asymptotically zero uniformly in the domain (4.19-h) as $\varepsilon$ tends to zero in $S_{j} \cap S_{j+1}$.

B. Estimate. Let $P_{j}(x, \varepsilon)$ and $P_{j+1}(x, \varepsilon)$ be the matrices given in Lemma 3.2. Then $P_{j}(x, \varepsilon) \Phi_{j h}(x, \varepsilon)$ and $P_{j+1}(x, \varepsilon) \Phi_{j+1, h}(x, \varepsilon)$ are two fundamental matrix solutions of (2.2) in the domain $x \in D_{1}, \varepsilon \in S_{j} \cap S_{j+1}$. Therefore, there exist two-by-two matrices $L_{h}(\varepsilon)$ such that

$$
P_{j+1}(x, \varepsilon) \Phi_{j+1, h}(x, \varepsilon)=P_{j}(x, \varepsilon) \Phi_{j h}(x, \varepsilon) L_{h}(\varepsilon) .
$$

Note that $L_{h}(\varepsilon)$ does not depend on $x$. It follows from (4.20-0) that

$$
\exp \left\{-\frac{x^{2} J}{2 \varepsilon}\right\} L_{0}(\varepsilon) \exp \left\{x^{2} J / 2 \varepsilon\right\}=Q_{j 0}(x, \varepsilon)^{-1} P_{j}(x, \varepsilon)^{-1} P_{j+1}(x, \varepsilon) Q_{j+1,0}(x, \varepsilon) .
$$

Hence, the matrix

$$
\exp \left\{-x^{2} J / 2 \varepsilon\right\} L_{0}(\varepsilon) \exp \left\{x^{2} J / 2 \varepsilon\right\}-I_{2}
$$

is asymptotically zero as $\varepsilon$ tends to zero in $S_{j} \cap S_{j+1}$ uniformly in the domain (4.19-0), where $I_{2}$ is the two-by-two identity matrix.

In the same way we can prove that the matrix

$$
\exp \left\{x^{2} J / 2 \varepsilon\right\} L_{1}(\varepsilon) \exp \left\{-x^{2} J / 2 \varepsilon\right\}-I_{2}
$$

is asymptotically zero as $\varepsilon$ tends to zero in $S_{j} \cap S_{j+1}$ uniformly in the domain (4.19-1).

From the connection formulas $(4.13-j),(4.20-0)$ and (4.20-1), we can derive

$$
L_{1}(\varepsilon)=M\left(p+\delta_{j}(\varepsilon)\right) L_{0}(\varepsilon) M\left(p+\delta_{j+1}(\varepsilon)\right)^{-1} .
$$

Set

$$
L_{0}(\varepsilon)=\left[\begin{array}{ll}
C_{11}(\varepsilon) & C_{12}(\varepsilon) \\
C_{21}(\varepsilon) & C_{22}(\varepsilon)
\end{array}\right], \quad L_{1}(\varepsilon)=\left[\begin{array}{ll}
\hat{C}_{11}(\varepsilon) & \hat{C}_{12}(\varepsilon) \\
\hat{C}_{21}(\varepsilon) & \hat{C}_{22}(\varepsilon)
\end{array}\right]
$$


Then from (4.23) and (4.24) we derive

(4.25)

$$
\begin{aligned}
\hat{C}_{11}(\varepsilon)= & C\left(p+\delta_{j}(\varepsilon)\right) C_{12}(\varepsilon)+C_{22}(\varepsilon), \\
\hat{C}_{12}(\varepsilon)= & \left\{C\left(p+\delta_{j}(\varepsilon)\right) C_{11}(\varepsilon)+C_{21}(\varepsilon)\right\} / \tilde{C}\left(p+\delta_{j+1}(\varepsilon)\right) \\
& -C\left(p+\delta_{j+1}(\varepsilon)\right)\left\{C\left(p+\delta_{j}(\varepsilon)\right) C_{12}(\varepsilon)+C_{22}(\varepsilon)\right\} / \tilde{C}\left(p+\delta_{j+1}(\varepsilon)\right), \\
\hat{C}_{21}(\varepsilon)= & \tilde{C}\left(p+\delta_{j}(\varepsilon)\right) C_{12}(\varepsilon), \\
\hat{C}_{22}(\varepsilon)= & \left\{\tilde{C}\left(p+\delta_{j}(\varepsilon)\right) C_{11}(\varepsilon)\right\} / \tilde{C}\left(p+\delta_{j+1}(\varepsilon)\right) \\
& -C\left(p+\delta_{j+1}(\varepsilon)\right)\left\{\tilde{C}\left(p+\delta_{j}(\varepsilon)\right) C_{12}(\varepsilon)\right\} / \tilde{C}\left(p+\delta_{j+1}(\varepsilon)\right) .
\end{aligned}
$$

Since

$$
\begin{aligned}
& \exp \left\{-x^{2} J / 2 \varepsilon\right\} L_{0}(\varepsilon) \exp \left\{x^{2} J / 2 \varepsilon\right\} \\
&=\left[\begin{array}{cc}
C_{11}(\varepsilon) & C_{12}(\varepsilon) \exp \left(-x^{2} / \varepsilon\right) \\
C_{21}(\varepsilon) \exp \left(x^{2} / \varepsilon\right) & C_{22}(\varepsilon)
\end{array}\right],
\end{aligned}
$$

and

$$
\begin{aligned}
& \exp \left\{x^{2} J / 2 \varepsilon\right\} L_{1}(\varepsilon) \exp \left\{-x^{2} J / 2 \varepsilon\right\} \\
&=\left[\begin{array}{cc}
\hat{C}_{11}(\varepsilon) & \hat{C}_{12}(\varepsilon) \exp \left(x^{2} / \varepsilon\right) \\
\hat{C}_{21}(\varepsilon) \exp \left(-x^{2} / \varepsilon\right) & \hat{C}_{22}(\varepsilon)
\end{array}\right],
\end{aligned}
$$

then, from (4.21) and (4.26), we have

$$
\left|C_{21}(\varepsilon)\right| \leqslant m_{21}\left|\exp \left(-x^{2} / \varepsilon\right)\right|,
$$

for some small positive number $m_{21}$, uniformly in the domain (4.19-0). Note that $\left|\arg \left(x / \varepsilon^{1 / 2}\right)-\frac{1}{4} \pi\right| \leqslant \frac{1}{2} \pi-\lambda$ if and only if $\frac{1}{2} \arg \varepsilon-\frac{1}{4} \pi+\lambda \leqslant \arg x \leqslant \frac{1}{2} \arg \varepsilon$ $+\frac{3}{4} \pi-\lambda$. If $\lambda$ is a sufficiently small positive number (e.g. $0<\lambda<\frac{1}{4} \pi-\frac{1}{2} \omega_{0}$ ), then

$$
\frac{1}{2} \arg \varepsilon-\frac{1}{4} \pi+\lambda<\frac{1}{2} \arg \varepsilon-\frac{1}{4} \pi+\frac{1}{4} \pi-\frac{1}{2} \omega_{0}<0,
$$

and

$$
\frac{1}{2} \arg \varepsilon+\frac{3}{4} \pi-\lambda>\frac{1}{2} \arg \varepsilon+\frac{3}{4} \pi-\frac{1}{4} \pi+\frac{1}{2} \omega_{0}>0 .
$$

Set $x=r_{0}$. Since $r_{0} \in D_{1}$ and $\arg \left(r_{0}\right)=0$ (note that $a<r_{0}<r_{2} \leqslant r_{1}$ ), then $r_{0}$ is in the domain (4.19-0) if $|\arg \varepsilon| \leqslant \omega_{0}$. Since (4.28) holds uniformly in the domain (4.19-0), we obtain

$$
\left|C_{21}(\varepsilon)\right| \leqslant m_{21} \exp \left\{-r_{0}^{2} \operatorname{Re}(1 / \varepsilon)\right\} \quad \text { for } \varepsilon \in S_{j} \cap S_{j+1} \text {. }
$$

Similarly, from (4.22) and (4.27), we have

$$
\left|\hat{C}_{12}(\varepsilon)\right| \leqslant \hat{m}_{12}\left|\exp \left(-x^{2} / \varepsilon\right)\right|
$$

for some small positive number $\hat{m}_{12}$, uniformly in the domain (4.19-1). Note that $\left|\arg \left(x / \varepsilon^{1 / 2}\right)-\frac{1}{4} \pi-\frac{1}{2} \pi\right| \leqslant \frac{1}{2} \pi-\lambda$ iff

$$
\frac{1}{2} \arg \varepsilon+\frac{1}{4} \pi+\lambda \leqslant \arg x \leqslant \frac{1}{2} \arg \varepsilon+\frac{5}{4} \pi-\lambda .
$$


Since

$$
\frac{1}{2} \arg \varepsilon+\frac{1}{4} \pi+\lambda<\frac{1}{2} \arg \varepsilon+\frac{1}{4} \pi+\frac{1}{4} \pi-\frac{1}{2} \omega_{0}<\frac{1}{2} \pi,
$$

and

$$
\frac{1}{2} \arg \varepsilon+\frac{5}{4} \pi-\lambda>\frac{1}{2} \arg \varepsilon+\frac{5}{4} \pi-\frac{1}{4} \pi+\frac{1}{2} \omega_{0}>\pi,
$$

$x=-r_{0}$ is in the domain (4.19-1), and we obtain

$$
\left|\hat{C}_{12}(\varepsilon)\right| \leqslant \hat{m}_{12} \exp \left\{-r_{0}^{2} \operatorname{Re}(1 / \varepsilon)\right\} \quad \text { for } \varepsilon \in S_{j} \cap S_{j+1} .
$$

Furthermore, as in Sibuya [14, p. 663], we obtain

$$
\left|C_{12}(\varepsilon)\right| \leqslant m_{12} \exp \left\{-r_{3}^{2} /|\varepsilon|\right\} \quad \text { for } \varepsilon \in S_{j} \cap S_{j+1},
$$

where $m_{12}$ is a positive number. It follows from (4.25) that

$$
\begin{gathered}
C\left(p+\delta_{j}(\varepsilon)\right) C_{11}(\varepsilon)-C\left(p+\delta_{j+1}(\varepsilon)\right)\left\{C\left(p+\delta_{j}(\varepsilon)\right) C_{12}(\varepsilon)+C_{22}(\varepsilon)\right\} \\
=\hat{C}_{12}(\varepsilon) \tilde{C}\left(p+\delta_{j+1}(\varepsilon)\right)-C_{21}(\varepsilon) .
\end{gathered}
$$

Since $C_{11}(\varepsilon)$ is asymptotically one as $\varepsilon$ tends to zero in $S_{j} \cap S_{j+1}$ (cf. (4.21) and (4.26)), we can derive (from (4.29) and (4.31))

$$
\begin{aligned}
& \mid C\left(p+\delta_{j}(\varepsilon)\right)- C\left(p+\delta_{j+1}(\varepsilon)\right)\left\{\frac{C_{22}(\varepsilon)}{C_{11}(\varepsilon)}+C\left(p+\delta_{j}(\varepsilon)\right) \frac{C_{12}(\varepsilon)}{C_{11}(\varepsilon)}\right\} \mid \\
& \leqslant m^{*} \exp \left\{-r_{0}^{2} \operatorname{Re}(1 / \varepsilon)\right\} \text { for } \varepsilon \in S_{j} \cap S_{j+1},
\end{aligned}
$$

for some positive number $m^{*}$. Let

$$
f_{j}(\varepsilon)=\frac{C_{22}(\varepsilon)}{C_{11}(\varepsilon)}+C\left(p+\delta_{j}(\varepsilon)\right) \frac{C_{12}(\varepsilon)}{C_{11}(\varepsilon)} .
$$

Then $f_{j}(\varepsilon)$ is holomorphic in $S_{j} \cap S_{j+1}$ and asymptotically one as $\varepsilon$ tends to zero in $S_{j} \cap S_{j+1}$ (cf. (4.21), (4.26) and (4.32)).

LEMMA 4.4. There exist functions $g_{j}(\varepsilon)$ in $S_{j}$ which are, respectively, holomorphic in $S_{j}$ and asymptotically one as $\varepsilon$ tends to zero in $S_{j}$ such that $f_{j}(\varepsilon)=g_{j+1}(\varepsilon) / g_{j}(\varepsilon)$ $(j=1,2, \ldots, \nu-1)$ in $S_{j} \cap S_{j+1}$.

REMARK. Here we replace $\rho$ by a smaller $\tilde{\rho}$.

We shall prove this lemma in $\S 4 \mathrm{D}$. By Lemma 4.4 we obtain

$$
\left|C\left(p+\delta_{j}(\varepsilon)\right) g_{j}(\varepsilon)-C\left(p+\delta_{j+1}(\varepsilon)\right) g_{j+1}(\varepsilon)\right| \leqslant m \exp \left\{-r_{0}^{2} \operatorname{Re}(1 / \varepsilon)\right\}
$$

in $S_{j} \cap S_{j+1}$ for some positive number $m$. Since $d C(p) / d \alpha \neq 0$ and $C(p)=0$ (cf. (4.9)), we have

$$
K_{1}\left|\delta_{j}(\varepsilon)\right| \leqslant\left|C\left(p+\delta_{j}(\varepsilon)\right)\right| \leqslant K_{2}\left|\delta_{j}(\varepsilon)\right|
$$

in $S_{j}$ for some positive numbers $K_{1}$ and $K_{2}$. Let

$$
\phi_{j}(\varepsilon)=C\left(p+\delta_{j}(\varepsilon)\right) g_{j}(\varepsilon) \text { for } \varepsilon \in S_{j} .
$$

Then from (4.33) we have

$$
\left|\phi_{j}(\varepsilon)-\phi_{j+1}(\varepsilon)\right| \leqslant m \exp \left\{-r_{0}^{2} \operatorname{Re}(1 / \varepsilon)\right\} \quad \text { in } S_{j} \cap S_{j+1} .
$$


Also, from (4.34), (4.3) and (4.4) we have

$$
\left|\phi_{1}(\varepsilon)\right| \leqslant \tilde{K} \exp \left\{-r_{0}^{2} \operatorname{Re}(1 / \varepsilon)\right\}
$$

on the line segment arg $=-\omega_{0}, 0<|\varepsilon|<\rho$, and

$$
\left|\phi_{\nu}(\varepsilon)\right| \leqslant \tilde{K} \exp \left\{-r_{0}^{2} \operatorname{Re}(1 / \varepsilon)\right\}
$$

on the line segment $\arg \varepsilon=\omega_{0}, 0<|\varepsilon|<\rho$ for some positive number $\tilde{K}$. Let $H_{1}=\max \{m, \tilde{K}\}$; by Theorem 3.3 we obtain

$$
\left|\phi_{j}(\varepsilon)\right| \leqslant H \exp \left\{-r_{0}^{2} \operatorname{Re}(1 / \varepsilon)\right\}
$$

for some positive number $H$ for $\varepsilon \in S_{j}, j=1,2, \ldots, \nu$. Thus, from (4.34) and (4.35) we have

$$
\left|\delta_{j}(\varepsilon)\right| \leqslant M_{1} \exp \left\{-r_{0}^{2} \operatorname{Re}(1 / \varepsilon)\right\}
$$

for some positive number $M_{1}$ for $\varepsilon \in S_{j}, j=1,2, \ldots, \nu$. This completes the proof of (4.1).

C. Resonance. In this section we shall prove Theorem 2.3 and derive the resonance from Theorem 2.3. To do this we shrink our domain $D_{1}$ so that $a<r_{2}<r_{0}$, where $r_{0}$ is the same number as in the last section, and choose $a$ sufficiently small positive number $\lambda$ such that $\cos \lambda>r_{2}^{2} / r_{0}^{2}$.

Let us recall that there is a transformation $W=P(x, \varepsilon) V$ which reduces (2.2) to

$$
\varepsilon V^{\prime}=\left[\begin{array}{cc}
0 & 1 \\
x^{2}-\varepsilon(p+\delta(\varepsilon)) & 0
\end{array}\right] V
$$

where $P(x, \varepsilon) \sim T(x, \varepsilon)$ uniformly in the domain $D_{1}$ as $\varepsilon$ tends to zero in

$$
\{\varepsilon:|\arg \varepsilon|<\lambda, 0<|\varepsilon|<M\} .
$$

In order to finish the proof of Theorem 2.3, we need to find a two-by-two matrix, say $B(x, \varepsilon)$, such that the transformation $V=B(x, \varepsilon) U$ reduces $(4.36)$ to

$$
\varepsilon U^{\prime}=\left[\begin{array}{cc}
0 & 1 \\
x^{2}-\varepsilon p & 0
\end{array}\right] U
$$

in the domain (4.37),

$$
D_{2}=D_{1} \backslash\left\{x=r e^{i \theta} \in D_{1}:-\frac{3}{4} \pi-\lambda \leqslant \theta \leqslant-\frac{1}{4} \pi+\lambda\right\} .
$$

Once this is done, we will choose $S(x, \varepsilon)$ in Theorem 2.3 as $S(x, \varepsilon)=P(x, \varepsilon) B(x, \varepsilon)$. Therefore, $B(x, \varepsilon)$ must be asymptotic to $I_{2}$ as $\varepsilon$ tends to zero in (4.37). Note that from the result in the last section, we already have

$$
|\delta(\varepsilon)| \leqslant M_{1} \exp \left\{-r_{0}^{2} \operatorname{Re}(1 / \varepsilon)\right\}
$$

for $\varepsilon$ in (4.37) for some positive number $M_{1}$. Theorem 2.3 is an immediate consequence of

THEOREM 4.5. Let $\lambda$ and $M$ be sufficiently small positive numbers such that $\cos \lambda>r_{2}^{2} / r_{0}^{2}$, and let (4.38) hold. Then there exists a two-by-two matrix $B(x, \varepsilon)$ whose components are holomorphic in two variables $x$ and $\varepsilon$ in the domain

$$
x \in D_{1}, \quad \varepsilon \in\{\varepsilon:|\arg \varepsilon|<\lambda, 0<|\varepsilon|<M\}
$$


such that:

(i) the matrix $B(x, \varepsilon) \sim I_{2}$ as $\varepsilon$ tends to zero in (4.37) uniformly in the domain

(ii) the transformation

$$
V=B(x, \varepsilon) U
$$

reduces (4.36) to (2.10) in the domain (4.40).

Proof. Set

$$
\begin{aligned}
& \Phi_{h}(x, \varepsilon)=\Lambda(\varepsilon) \Psi_{h}\left(x / \varepsilon^{1 / 2}, p+\delta(\varepsilon)\right) \\
& \tilde{\Phi}_{h}(x, \varepsilon)=\Lambda(\varepsilon) \Psi_{h}\left(x / \varepsilon^{1 / 2}, p\right)
\end{aligned}
$$

(cf. (4.8-0) and (4.8-1)). Then $\Phi_{h}(x, \varepsilon)$ and $\tilde{\Phi}_{h}(x, \varepsilon)$ are fundamental matrix solutions of (4.36) and (2.10), respectively, such that

$$
\Phi_{0}(x, \varepsilon)=\Phi_{1}(x, \varepsilon) M(p+\delta(\varepsilon)) \quad \text { and } \quad \tilde{\Phi}_{0}(x, \varepsilon)=\tilde{\Phi}_{1}(x, \varepsilon) M(p)
$$

where $M$ is given by (4.11). Set

$$
B(x, \varepsilon)=\Phi_{0}(x, \varepsilon) \tilde{\Phi}_{0}(x, \varepsilon)^{-1} .
$$

Then the transformation $V=B(x, \varepsilon) U$ reduces (4.38) to (2.10). Therefore, the main part of the proof is to show that $B(x, \varepsilon)-I_{2}$ is asymptotically zero as $\varepsilon$ tends to zero in (4.37) uniformly in the domain (4.38).

Set $J=\left[\begin{array}{cc}1 & 0 \\ 0 & -1\end{array}\right]$ and

$$
\left\{\begin{array}{l}
Q_{h}(x, \varepsilon)=\Phi_{h}(x, \varepsilon) \exp \left\{(-1)^{h} x^{2} J / 2 \varepsilon\right\} \\
\tilde{Q}_{h}(x, \varepsilon)=\tilde{\Phi}_{h}(x, \varepsilon) \exp \left\{(-1)^{h} x^{2} J / 2 \varepsilon\right\}
\end{array} \quad(h=0,1) .\right.
$$

By Lemmas 4.2 and 4.3 we know that if $\varepsilon$ is in (4.37) and

$$
x \in D_{1}, \quad\left|\arg x-\frac{1}{4} \pi-\frac{1}{2} h \pi\right| \leqslant \frac{1}{2} \pi-\lambda,
$$

where $\lambda$ is a small positive number, then we have

$$
\begin{cases}\left\|Q_{h}(x, \varepsilon)\right\| \leqslant \tilde{c}|\varepsilon|^{q}, & \left\|Q_{h}(x, \varepsilon)^{-1}\right\| \leqslant \tilde{c}|\varepsilon|^{q}, \\ \left\|\tilde{Q}_{h}(x, \varepsilon)\right\| \leqslant \tilde{c}|\varepsilon|^{q}, & \left\|\tilde{Q}_{h}(x, \varepsilon)^{-1}\right\| \leqslant \tilde{c}|\varepsilon|^{q}\end{cases}
$$

where $\tilde{c}$ is a positive number depending on $\lambda$ and $q$ is a real number. Furthermore, the matrix

$$
Q_{h}(x, \varepsilon)-\tilde{Q}_{h}(x, \varepsilon)
$$

is asymptotically zero as $\varepsilon$ tends to zero in (4.37) uniformly in (4.42-h).

Since $\Phi_{1}(x, \varepsilon)$ and $B(x, \varepsilon) \tilde{\Phi}_{1}(x, \varepsilon)$ are two fundamental matrix solutions of (4.36) for $x \in D_{1}$ and $\varepsilon$ is in (4.37), there exists a two-by-two matrix $\tilde{L}_{1}(\varepsilon)$ such that

$$
\Phi_{1}(x, \varepsilon) \tilde{L}_{1}(\varepsilon)=B(x, \varepsilon) \tilde{\Phi}_{1}(x, \varepsilon) .
$$


Note that $\tilde{L}_{1}(\varepsilon)$ does not depend on $x$, and

$$
\begin{aligned}
\tilde{L}_{1}(\varepsilon) & =\Phi_{1}(x, \varepsilon)^{-1} B(x, \varepsilon) \tilde{\Phi}_{1}(x, \varepsilon) \\
& =M(p+\delta(\varepsilon)) \Phi_{0}(x, \varepsilon)^{-1} B(x, \varepsilon) \tilde{\Phi}_{0}(x, \varepsilon) M(p)^{-1} \\
& =M(p+\delta(\varepsilon)) M(p)^{-1} .
\end{aligned}
$$

We have

$$
\begin{aligned}
M(p+\delta(\varepsilon)) M(p)^{-1}-I_{2} & =\left[\begin{array}{cc}
C(p+\delta(\varepsilon)) & 1 \\
\tilde{C}(p+\delta(\varepsilon)) & 0
\end{array}\right] \cdot\left[\begin{array}{cc}
0 & 1 / \tilde{C}(p) \\
1 & -C(p) / \tilde{C}(p)
\end{array}\right]-\left[\begin{array}{ll}
1 & 0 \\
0 & 1
\end{array}\right] \\
& =\left[\begin{array}{ll}
0 & \{C(p+\delta(\varepsilon))-C(p)\} / \tilde{C}(p) \\
0 & \{\tilde{C}(p+\delta(\varepsilon))-\tilde{C}(p)\} / \tilde{C}(p)
\end{array}\right] \\
& =\left[\begin{array}{ll}
0 & O(\delta(\varepsilon)) \\
0 & O(\delta(\varepsilon))
\end{array}\right] \text { for }|\arg \varepsilon|<\lambda, 0<|\varepsilon|<M
\end{aligned}
$$

Furthermore,

$$
\begin{aligned}
B(x, \varepsilon) & =\Phi_{0}(x, \varepsilon) \tilde{\Phi}_{0}(x, \varepsilon)^{-1} \\
& =Q_{0}(x, \varepsilon) \exp \left\{-x^{2} J / 2 \varepsilon\right\} \exp \left\{x^{2} J / 2 \varepsilon\right\} \tilde{Q}_{0}(x, \varepsilon)^{-1} \\
& =Q_{0}(x, \varepsilon) \tilde{Q}_{0}(x, \varepsilon)^{-1} .
\end{aligned}
$$

It follows from (4.44-0) that $B(x, \varepsilon)-I_{2}$ is asymptotically zero as $\varepsilon$ tends to zero in (4.37) uniformly in the domain (4.42-0). Also, from (4.45) we have

$$
\begin{aligned}
& B(x, \varepsilon)=\Phi_{1}(x, \varepsilon) \tilde{L}_{1}(\varepsilon) \tilde{\Phi}_{1}(x, \varepsilon)^{-1} \\
& =Q_{1}(x, \varepsilon) \exp \left(x^{2} J / 2 \varepsilon\right) \tilde{L}_{1}(\varepsilon) \exp \left(-x^{2} J / 2 \varepsilon\right) \tilde{Q}_{1}(x, \varepsilon)^{-1} \\
& =Q_{1}(x, \varepsilon) \cdot\left[\begin{array}{cc}
\exp \left(x^{2} / 2 \varepsilon\right) & 0 \\
0 & \exp \left(-x^{2} / 2 \varepsilon\right)
\end{array}\right]\left[\begin{array}{cc}
1 & O(\delta(\varepsilon)) \\
0 & 1+O(\delta(\varepsilon))
\end{array}\right] \\
& \times \exp \left(-x^{2} J / 2 \varepsilon\right) \tilde{Q}_{1}(x, \varepsilon)^{-1} \\
& =Q_{1}(x, \varepsilon) \cdot\left[\begin{array}{cc}
\exp \left(x^{2} / 2 \varepsilon\right) & \left\{\exp \left(x^{2} / 2 \varepsilon\right)\right\} O(\delta(\varepsilon)) \\
0 & \exp \left(-x^{2} / 2 \varepsilon\right)(1+O(\delta(\varepsilon)))
\end{array}\right] \\
& \times\left[\begin{array}{cc}
\exp \left(-x^{2} / 2 \varepsilon\right) & 0 \\
0 & \exp \left(x^{2} / 2 \varepsilon\right)
\end{array}\right] \cdot \tilde{Q}_{1}(x, \varepsilon)^{-1} \\
& =Q_{1}(x, \varepsilon) \cdot\left[\begin{array}{cc}
1 & \left\{\exp \left(x^{2} / \varepsilon\right)\right\} O(\delta(\varepsilon)) \\
0 & 1+O(\delta(\varepsilon))
\end{array}\right] \cdot \tilde{Q}_{1}(x, \varepsilon)^{-1} \\
& =Q_{1}(x, \varepsilon)\left\{I_{2}+\left[\begin{array}{cc}
0 & \exp \left(x^{2} / \varepsilon\right) O(\delta(\varepsilon)) \\
0 & O(\delta(\varepsilon))
\end{array}\right]\right\} \tilde{Q}_{1}(x, \varepsilon)^{-1} \\
& =Q_{1}(x, \varepsilon) \tilde{Q}_{1}(x, \varepsilon)^{-1}+Q_{1}(x, \varepsilon)\left[\begin{array}{cc}
0 & \left\{\exp \left(x^{2} / \varepsilon\right)\right\} O(\delta(\varepsilon)) \\
0 & O(\delta(\varepsilon))
\end{array}\right] \tilde{Q}_{1}(x, \varepsilon)^{-1},
\end{aligned}
$$


as $\varepsilon$ tends to zero in (4.37). Since

$$
Q_{1}(x, \varepsilon)-\tilde{Q}_{1}(x, \varepsilon)
$$

is asymptotically zero as $\varepsilon$ tends to zero in (4.37) uniformly in (4.42-1), then there exists a two-by-two matrix $F_{1}(x, \varepsilon)$ such that $Q_{1}(x, \varepsilon)=\tilde{Q}_{1}(x, \varepsilon)+F_{1}(x, \varepsilon)$ and $F_{1}(x, \varepsilon)$ is asymptotically zero as $\varepsilon$ tends to zero in (4.37) uniformly in (4.42-1). Thus,

$$
\begin{aligned}
B(x, \varepsilon)-I_{2}= & F_{1}(x, \varepsilon) \tilde{Q}_{1}(x, \varepsilon)^{-1} \\
& +Q_{1}(x, \varepsilon)\left[\begin{array}{cc}
0 & \left\{\exp \left(x^{2} / \varepsilon\right)\right\} O(\delta(\varepsilon)) \\
0 & O(\delta(\varepsilon))
\end{array}\right] \tilde{Q}_{1}(x, \varepsilon)^{-1} .
\end{aligned}
$$

Since $\delta(\varepsilon)$ satisfies (4.39) for $|\arg \varepsilon|<\lambda, 0<|\varepsilon|<M$, we have

(i) $\delta(\varepsilon)$ is asymptotically zero as $\varepsilon$ tends to zero in (4.37).

Also, if $x$ is in (4.42-1) and $\varepsilon$ in (4.37) then

$$
\begin{aligned}
\left|\delta(\varepsilon) \exp \left(x^{2} / \varepsilon\right)\right| & \leqslant M_{1} \exp \left\{-r_{0}^{2} \operatorname{Re}(1 / \varepsilon)\right\} \exp \left\{r_{2}^{2} /|\varepsilon|\right\} \\
& =M_{1} \exp \left\{-r_{0}^{2} \operatorname{Re}(1 / \varepsilon)+r_{2}^{2} /|\varepsilon|\right\} \\
& =M_{1} \exp \left\{-|\varepsilon|^{-1}\left[r_{0}^{2} \cos (|\arg \varepsilon|)-r_{2}^{2}\right]\right\}
\end{aligned}
$$

since $|\arg \varepsilon|<\lambda<\frac{1}{2} \pi$ implies that $\cos (|\arg \varepsilon|)>\cos \lambda>r_{2}^{2} / r_{0}^{2}$.

Hence, we have

(ii) $\delta(\varepsilon) \exp \left(x^{2} / \varepsilon\right)$ is asymptotically zero as $\varepsilon$ tends to zero in (4.37) uniformly in $(4.42-1)$.

Utilizing (i), (ii) and the fact that $F_{1}(x, \varepsilon)$ is asymptotically zero as $\varepsilon$ tends to zero in (4.37) uniformly in (4.42-1), together with (4.43-1), we obtain $B(x, \varepsilon)-I_{2}$ is asymptotically zero as $\varepsilon$ tends to zero in (4.37) uniformly in the domain (4.42-1). The domain $D_{2}$ is the union of (4.42-0) and (4.42-1). Thus we complete the proof of Theorem 4.5 .

Now we shall derive the resonance from Theorem 2.3. (2.10) is equivalent to

$$
\varepsilon^{2} u^{\prime \prime}-\left(x^{2}-\varepsilon p\right) u=0
$$

which admits a solution

$$
u(x, \varepsilon)=C w_{1}\left(x / \varepsilon^{1 / 2}, p\right),
$$

where $C$ is an arbitrary constant. Since $(p-1) / 2$ is a nonnegative integer,

$$
u(x, \varepsilon)=C e^{-x^{2} / 2 \varepsilon}\left(\frac{x}{\varepsilon^{1 / 2}}\right)^{(p-1) / 2}\left\{1+\alpha_{1} \frac{\varepsilon}{x^{2}}+\alpha_{2} \frac{\varepsilon^{2}}{x^{4}}+\cdots+\alpha_{\nu} \frac{\varepsilon^{\nu}}{x^{2 \nu}}\right\},
$$

where $\alpha_{1}, \ldots, \alpha_{\nu}$ are constants, $\nu$ is a positive integer such that $2 \nu \leqslant(p-1) / 2$, and

$$
\varepsilon u^{\prime}(x, \varepsilon)=C \sqrt{\varepsilon} e^{-x^{2} / 2 \varepsilon}\left(\frac{x}{\varepsilon^{1 / 2}}\right)^{(p+1) / 2}\left\{-1+\beta_{1} \frac{\varepsilon}{x^{2}}+\beta_{2} \frac{\varepsilon^{2}}{x^{4}}+\cdots+\beta_{\nu} \frac{\varepsilon^{\nu}}{x^{2 \nu}}\right\},
$$

where $\beta_{1}, \ldots, \beta_{\nu}$ are constants (cf. Hermite polynomial in [17]). Thus,

$$
U=\left[\begin{array}{c}
u \\
\varepsilon u^{\prime}
\end{array}\right]
$$


is a solution of (2.10). Set

$$
S(x, \varepsilon)=\left[\begin{array}{ll}
S_{11}(x, \varepsilon) & S_{12}(x, \varepsilon) \\
S_{21}(x, \varepsilon) & S_{22}(x, \varepsilon)
\end{array}\right]
$$

in Theorem 2.3. Then $S(x, \varepsilon) \sim T(x, \varepsilon)$ as $\varepsilon$ tends to zero in (4.37) uniformly in $D_{2}$, and

$$
w(x, \varepsilon)=S_{11}(x, \varepsilon) u(x, \varepsilon)+S_{12}(x, \varepsilon) \varepsilon u^{\prime}(x, \varepsilon)
$$

is a solution of

$$
\varepsilon^{2} w^{\prime \prime}-\left(x^{2}+\varepsilon R(x, \varepsilon)\right) w=0
$$

Hence

$$
y(x, \varepsilon)=\exp \left\{-\frac{1}{2 \varepsilon} \int_{0}^{x}\left[-2 \tau+\varepsilon f_{1}(\tau, \varepsilon)\right] d \tau\right\} w
$$

is a solution of $(1.1)$, where $f_{1}(\tau, \varepsilon)$ is holomorphic in $\tau \in D_{1},|\varepsilon|<\rho$. It follows from (4.47)-(4.49) that

$$
\begin{aligned}
y(x, \varepsilon)= & C \varepsilon^{-(p-1) / 4} \exp \left\{-\frac{1}{2} \int_{0}^{x} f_{1}(\tau, \varepsilon) d \tau\right\} \\
& \times\left\{S_{11}(x, \varepsilon)\left[x^{(p-1) / 2}+\alpha_{1} \varepsilon x^{(p-5) / 2}+\cdots+\alpha_{\nu} \varepsilon^{\nu} x^{q_{\nu}}\right]\right. \\
& \left.\quad+x S_{12}(x, \varepsilon)\left[-x^{(p-1) / 2}+\beta_{1} \varepsilon x^{(p-5) / 2}+\cdots+\beta_{\nu} \varepsilon^{\nu} x^{q_{\nu}}\right]\right\}
\end{aligned}
$$

where $q_{\nu}$ is a nonnegative integer. Set $C=\varepsilon^{(p-1) / 4}$. Then (4.50) becomes

$$
\begin{aligned}
y(x, \varepsilon)= & \exp \left\{-\frac{1}{2} \int_{0}^{x} f_{1}(\tau, \varepsilon) d \tau\right\} \\
\times\{ & S_{11}(x, \varepsilon)\left[x^{(p-1) / 2}+\alpha_{1} \varepsilon x^{(p-5) / 2}+\cdots+\alpha_{\nu} \varepsilon^{\nu} x^{q_{\nu}}\right] \\
& \left.\quad+x S_{12}(x, \varepsilon)\left[-x^{(p-1) / 2}+\beta_{1} \varepsilon x^{(p-5) / 2}+\cdots+\beta_{\nu} \varepsilon^{\nu} x^{q_{\nu}}\right]\right\},
\end{aligned}
$$

and

$$
\lim _{\varepsilon \rightarrow 0} y(x, \varepsilon)=\left\{\exp \left[-\frac{1}{2} \int_{0}^{x} f_{1}(\tau, 0) d \tau\right]\right\}\left\{A_{0}(x)-x B(x)\right\} x^{(p-1) / 2}
$$

uniformly in $x \in \bar{D}_{2}$. Since $A_{0}(x)^{2}-(x B(x))^{2}=1$ in $D_{1}, \lim _{\varepsilon \rightarrow 0} y(x, \varepsilon)$ is a nontrivial solution of $f(x, 0) y^{\prime}+g(x, 0) y=0$.

D. Proof of the lemma. We will prove Lemma 4.4.

Proof. Since $f_{1}(\varepsilon)$ is holomorphic in $S_{1} \cap S_{2}$ and asymptotically one in $S_{1} \cap S_{2}$, then $\log f_{1}(\varepsilon)$ is holomorphic in $\tilde{S}_{1} \cap \tilde{S}_{2}$ and asymptotically zero in $\tilde{S}_{1} \cap \tilde{S}_{2}$, where $\tilde{S}_{j}=\left\{\varepsilon: a_{j}<\arg \varepsilon<b_{j}, 0<|\varepsilon|<\tilde{\rho}\right\}$ with some $\tilde{\rho}$ such that $0<\tilde{\rho}<\rho$. For given $\theta>0$, set

$$
V_{1}=\left\{\varepsilon: a_{2}+\theta<\arg \varepsilon<b_{1}-\theta, 0<|\varepsilon|<\tilde{\rho}\right\} .
$$

Then $V_{1} \subset \tilde{S}_{1} \cap \tilde{S}_{2}$ and $\gamma_{1}-\gamma_{1}^{*}$ are boundaries of $V_{1}$, where

$$
\gamma_{1}=\left\{\varepsilon: \varepsilon=t e^{i\left(a_{2}+\theta\right)}, 0<t<\tilde{\rho}\right\} \cup\left\{\varepsilon: \varepsilon=\tilde{\rho} e^{i \xi}, a_{2}+\theta \leqslant \xi \leqslant\left(b_{1}-a_{2}\right) / 2\right\}
$$


and

$$
\gamma_{1}^{*}=\left\{\varepsilon: \varepsilon=t e^{i\left(b_{1}-\theta\right)}, 0<t<\tilde{\rho}\right\} \cup\left\{\varepsilon: \varepsilon=\tilde{\rho} e^{-i \xi},\left(b_{1}-a_{2}\right) / 2 \leqslant \xi \leqslant b_{1}-\theta\right\} .
$$

By the Cauchy integral formula, we have

$$
\log f_{1}(\varepsilon)=\frac{1}{2 \pi i} \int_{\gamma_{1}} \frac{\log f_{1}(z)}{z-\varepsilon} d z-\frac{1}{2 \pi i} \int_{\gamma_{1}^{*}} \frac{\log f_{1}(z)}{z-\varepsilon} d z .
$$

Set

$$
\tilde{h}_{1}(\varepsilon)=\frac{1}{2 \pi i} \int_{\gamma_{1}} \frac{\log f_{1}(z)}{z-\varepsilon} d z, \quad h_{1}(\varepsilon)=\frac{1}{2 \pi i} \int_{\gamma_{1}^{*}} \frac{\log f_{1}(z)}{z-\varepsilon} d z
$$

Since

$$
\frac{1}{z-\varepsilon}=\sum_{m=0}^{N} z^{-(m+1)} \varepsilon^{m}+\frac{\varepsilon^{N+1}}{z^{N+1}(z-\varepsilon)}, \text { for all } N=0,1, \ldots,
$$

we have

$$
\tilde{h}_{1}(\varepsilon)=\sum_{m=0}^{N}\left[\frac{1}{2 \pi i} \int_{\gamma_{1}} z^{-(m+1)} \log f_{1}(z) d z\right] \varepsilon^{m}+\frac{\varepsilon^{N+1}}{2 \pi i} \int_{\gamma_{1}} \frac{\log f_{1}(z) d z}{z^{N+1}(z-\varepsilon)}
$$

and

$$
\left|\frac{\varepsilon}{2 \pi i} \int_{\gamma_{1}} \frac{\log f_{1}(z)}{z^{N+1}(z-\varepsilon)} d z\right| \rightarrow 0 \quad \text { as } \varepsilon \rightarrow 0
$$

Therefore

$$
\tilde{h}_{1}(\varepsilon) \sim \sum_{n=0}^{\infty} \tilde{p}_{n} \varepsilon^{n}
$$

as $\varepsilon$ tends to zero in $\hat{S}_{2}$, where

$$
\hat{S}_{1}=\left\{\varepsilon: a_{1}<\arg \varepsilon<b_{1}-\theta, 0<|\varepsilon|<\tilde{\beta}\right\} \subset \tilde{S}_{1},
$$

and

$$
\hat{S}_{2}=\left\{\varepsilon: a_{2}+\theta<\arg \varepsilon<b_{2}, 0<|\varepsilon|<\tilde{\beta}\right\} \subset \tilde{S}_{2} .
$$

Note that $\tilde{h}_{1}(\varepsilon)$ is also holomorphic in $\hat{S}_{2}$. Similarly,

$$
h_{1}(\varepsilon) \sim \sum_{n=0}^{\infty} p_{n} \varepsilon^{n}
$$

as $\varepsilon$ tends to zero in $\hat{S}_{1}$, and $h_{1}(\varepsilon)$ is holomorphic in $\hat{S}_{1}$. Also, $\log f_{1}(\varepsilon)=\tilde{h}_{1}(\varepsilon)-h_{1}(\varepsilon)$. Thus,

$$
f_{1}(\varepsilon)=\tilde{H}_{1}(\varepsilon) / H_{1}(\varepsilon)
$$

where $H_{1}(\varepsilon)=\exp \left\{h_{1}(\varepsilon)\right\}$ and $\tilde{H}_{1}(\varepsilon)=\exp \left\{\tilde{h}_{1}(\varepsilon)\right\}$ are holomorphic in $\hat{S}_{1}$ and $\hat{S}_{2}$, respectively. Furthermore,

$$
H_{1}(\varepsilon) \sim \sum_{n=0}^{\infty} q_{n} \varepsilon^{n}
$$


as $\varepsilon$ tends to zero in $\hat{S}_{1}$ and

$$
\tilde{H}_{1}(\varepsilon) \sim \sum_{n=0}^{\infty} q_{n} \varepsilon^{n}
$$

as $\varepsilon$ tends to zero in $\hat{S}_{2}$. Since $f_{1}(\varepsilon)$ is asymptotically one as $\varepsilon$ tends to zero in $\hat{S}_{1} \cap \hat{S}_{2}$ and $f_{1}(\varepsilon) H_{1}(\varepsilon)=\tilde{H}_{1}(\varepsilon)$ in $\hat{S}_{1} \cap \hat{S}_{2}$, we have $q_{n}=\tilde{q}_{n}$ for all $n=0,1,2, \ldots$

By a theorem of J. F. Ritt (cf. W. Wasow [15]) there exists a function $F_{1}(\varepsilon)$ which is holomorphic in $\hat{S}_{1} \cup \hat{S}_{2}$, where $F_{1}(\varepsilon) \sim \sum_{n=0}^{\infty} q_{n} \varepsilon^{n}$ as $\varepsilon$ tends to zero in $\hat{S}_{1} \cup \hat{S}_{2}$. Set

$$
G_{1}(\varepsilon)=H_{1}(\varepsilon) / F_{1}(\varepsilon), \quad \tilde{G}_{1}(\varepsilon)=\tilde{H}_{1}(\varepsilon) / F_{1}(\varepsilon) .
$$

Then $G_{1}(\varepsilon)$ and $\tilde{G}_{1}(\varepsilon)$ are holomorphic in $\hat{S}_{1}$ and $\hat{S}_{2}$ and asymptotically one as $\varepsilon$ tends to zero in $\hat{S}_{1}$ and $\hat{S}_{2}$, respectively. Since $f_{1}(\varepsilon) G_{1}(\varepsilon)=\tilde{G}_{1}(\varepsilon)$, by continuation, we obtain $G_{1}(\varepsilon)$ and $\tilde{G}_{1}(\varepsilon)$ are holomorphic in $\tilde{S}_{1}$ and $\tilde{S}_{2}$ and asymptotically one as $\varepsilon$ tends to zero in $\tilde{S}_{1}$ and $\tilde{S}_{2}$, respectively. Since $\tilde{S}_{2} \cap \tilde{S}_{3}=\left(\tilde{S}_{1} \cup \tilde{S}_{2}\right) \cap \tilde{S}_{3}$ and $f_{2}(\varepsilon) \tilde{G}_{1}(\varepsilon)$ is holomorphic in $\tilde{S}_{2} \cap \tilde{S}_{3}$ and asymptotically one as $\varepsilon$ tends to zero in $\tilde{S}_{2} \cap \tilde{S}_{3}$, there exist $P_{1}(\varepsilon)$ and $Q_{1}(\varepsilon)$ holomorphic in $\tilde{S}_{1} \cup \tilde{S}_{2}$ and $\tilde{S}_{3}$ and asymptotically one as $\varepsilon$ tends to zero in $\tilde{S}_{1} \cup \tilde{S}_{2}$ and $\tilde{S}_{3}$, respectively, such that

$$
f_{2}(\varepsilon) \tilde{G}_{1}(\varepsilon)=Q_{1}(\varepsilon) / P_{1}(\varepsilon) \text {. }
$$

Then

$$
f_{2}(\varepsilon)=Q_{1}(\varepsilon) / P_{1}(\varepsilon) \tilde{G}_{1}(\varepsilon)
$$

Set

$$
f_{1}(\varepsilon)=P_{1}(\varepsilon) \tilde{G}_{1}(\varepsilon) / P_{1}(\varepsilon) G_{1}(\varepsilon)
$$

Note that $P_{1}(\varepsilon) G_{1}(\varepsilon)$ and $P_{1}(\varepsilon) \tilde{G}_{1}(\varepsilon)$ are holomorphic in $\tilde{S}_{1}$ and $\tilde{S}_{2}$ and asymptotically one as $\varepsilon$ tends to zero in $\tilde{S}_{1}$ and $\tilde{S}_{2}$, respectively. Continuing in this fashion, in a finite number of steps the resultant will meet the requirement of Lemma 4.4.

Acknowledgement. I would like to thank my advisor Professor Sibuya for his many valuable suggestions.

\section{REFERENCES}

1. M. Abramowitz and I. A. Stegun, Eds., Handbook of mathematical functions, Nat. Bur. Standards Appl. Math. Ser. 55, U. S. Government Printing Office, Washington, D.C., 1964.

2. R. C. Ackerberg and R. E. O'Malley, Boundary layer problems exhibiting resonance, Stud. Appl. Math. 49 (1970), 61-73.

3. L. P. Cook and W. Eckhaus, Resonance in a boundary value problem of singular perturbation type, Stud. Appl. Math. 52 (1973), 129-139.

4. P. F. Hsieh and Y. Sibuya, On the asymptotic integration of second order linear ordinary differential equations with polynomial coefficients, J. Math. Anal. Appl. 16 (1966), 84-103.

5. N. Kopell, A geometric approach to boundary layer problems exhibiting resonance, SIAM J. Appl. Math. 37 (1979), 436-458.

6. H. O. Kreiss and S. V. Parter, Remarks on singular perturbations with turning points, SIAM J. Math. Anal. 5 (1974), 230-251.

7. C-H. Lin, Phragmen-Lindelof theorem in a cohomological form, Univ. of Minnesota Math. Report 81-151.

8. The sufficiency of Matkowsky-condition in the problem of resonance, Ph.D. Thesis, Univ. of Minnesota, June 1982.

9. B. J. Matkowsky, On boundary layer problems exhibiting resonance, SIAM Rev. 17 (1975), 82-100. 
10. F. W. Olver, Sufficient conditions for Ackerber-O'Malley resonance, SIAM J. Math. Anal. 9 (1978), $328-355$

11. R. E. O’Malley, Introduction to singular perturbation, Academic Press, New York, 1974.

12. Y. Sibuya, Uniform simplification in a full neighborhood of a turning point, Mem. Amer. Math. Soc. No. 149 (1974).

13. Global theory of a second order linear ordinary differential equation with a polynomial coefficient, North-Holland, Amsterdam, 1975.

14. $\quad$ A theorem concerning uniform simplification at a transition point and the problem of resonance, SIAM J. Math. Anal. 12 (1981), 653-668.

15. W. Wasow, Asymptotic expansions for ordinary differential equations, Interscience, New York, 1965.

16. A. M. Watts, $A$ singular perturbation problem with a turning point, Bull. Austral. Math. Soc. 5 (1971), $61-73$.

17. E. T. Whittaker and G. H. Watson, $A$ course of modern analysis, 4th ed., Cambridge Univ. Press, Cambridge, 1952.

Department of Mathematics, University of Minnesota, Minneapolis, Minnesota 55455

Current address: Department of Mathematics, Fu Jen University, Hsinchuang 242, Taipei, Taiwan 